\title{
Integrability by quadratures of Hamiltonian systems and Picard-Fuchs type equations: The modern differential-geometric aspects
}

\author{
Y. A. Prykarpatsky, A. M. Samoilenko, D. L. \\ Blackmore, and A. K. Prykarpatsky
}




\title{
INTEGRABILITY BY QUADRATURES OF HAMILTONIAN SYSTEMS AND PICARD-FUCHS TYPE EQUATIONS: THE MODERN DIFFERENTIAL-GEOMETRIC ASPECTS
}

\author{
Y. A. PRYKARPATSKY, A. M. SAMOILENKO, D. L. BLACKMORE, \\ AND A. K. PRYKARPATSKY
}

This paper is dedicated to the 95-th birthday anniversary (1909-1993) of the greatest mathematician and physicist of the late 20th century, our teacher and academician Nikolay N. Bogoliubov

[Received: January 18, 2003 and, in revised form, July 15, 2004]

\begin{abstract}
Aвstract. There is developed a symplectic theory approach for partially solving the problem of algebraic-analytical construction of integral submanifold imbeddings for integrable via the abelian and nonabelian Liouville-Arnold theorems for Hamiltonian systems on canonically symplectic phase spaces. The fundamental role of so-called Picard-Fuchs type equations is revealed and their differentialgeometric and algebraic properties are studied in detail. Some interesting examples of integrable Hamiltonian systems demonstrating the algorithm of investigating the integral submanifolds imbedding mapping are studied in detail.

Mathematics Subject Classification: Primary 34A30, 34B01 Secondary 34B15

Keywords: Liouville-Arnold integrability by quadratures, Mishchenko-Fomenko noncommutative integrability, Hamiltonian dynamical systems, Picard-Fuchs equations, integral submanifold imbedding problem, hyperelliptic algebraic curves, symplectic structures, Koelerian structures
\end{abstract}

\section{INTRODUCTION}

As it is well-known [1,4], the integrability by quadratures of a differential equation in space $\mathbb{R}^{n}$ is a method of seeking its solutions by means of a finite number of algebraic operations (together with the inversion of functions) and "quadratures", i. e., calculation of integrals of known functions.

Assume that our differential equation is given as a Hamiltonian dynamical system on some appropriate symplectic manifold $\left(M^{2 n}, \omega^{(2)}\right), n \in \mathbb{Z}_{+}$, in the form

$$
d u / d t=\{H, u\},
$$

The first author was supported in part by a local AGH grant. 
where $u \in M^{2 n}, H: M^{2 n} \rightarrow \mathbb{R}$ is a sufficiently smooth Hamiltonian function [1,4] with respect to the Poisson bracket $\{\cdot, \cdot\}$ on $\mathcal{D}\left(M^{2 n}\right)$, dual to the symplectic structure $\omega^{(2)} \in \Lambda^{2}\left(M^{2 n}\right)$, and $t \in \mathbb{R}$ is the evolution parameter. More than one hundred and fifty years ago French mathematicians and physicists, first E. Bour and next J. Liouville, proved the first "integrability by quadratures" theorem which in modern terms [33] can be formulated as follows.

Theorem 1. Let $M^{2 n} \simeq T^{*}\left(\mathbb{R}^{n}\right)$ be a canonically symplectic phase space and there be given a dynamical system (1.1) with a Hamiltonian function $H: M^{2 n} \times \mathbb{R}_{t} \rightarrow \mathbb{R}$, possessing a Poissonian Lie algebra $\mathcal{G}$ of $n \in \mathbb{Z}_{+}$invariants $H_{j}: M^{2 n} \times \mathbb{R}_{t} \rightarrow \mathbb{R}$, $j=\overline{1, n}$, such that

$$
\left\{H_{i}, H_{j}\right\}=\sum_{s=1}^{n} c_{i j}^{s} H_{s},
$$

and for all $i, j, k=\overline{1, n}$ the $c_{i j}^{s} \in \mathbb{R}$ are constants on $M^{2 n} \times \mathbb{R}_{t}$. Suppose further that

$$
M_{h}^{n+1}:=\left\{(u, t) \in M \times \mathbb{R}_{t}: h\left(H_{j}\right)=h_{j}, j=\overline{1, n}, h \in \mathcal{G}^{*}\right\},
$$

the integral submanifold of the set $\mathcal{G}$ of invariants at a regular element $h \in \mathcal{G}^{*}$ is a well defined connected submanifold of $M \times \mathbb{R}_{t}$. Then, if:

(i) All functions of $\mathcal{G}$ are functionally independent on $M_{h}^{n+1}$;

(ii) $\sum_{s=1}^{n} c_{i j}^{s} h_{s}=0$ for all $i, j=\overline{1, n}$;

(iii) The Lie algebra $\mathcal{G}=\operatorname{span}_{\mathbb{R}}\left\{H_{j}: M^{2 n} \times \mathbb{R}_{t} \rightarrow \mathbb{R}: j=\overline{1, n}\right\}$ is solvable, the Hamiltonian system (1.1) on $M^{2 n}$ is integrable by quadratures.

As a simple corollary of the Bour-Liouville theorem one gets the following

Corollary 1. If a Hamiltonian system on $M^{2 n}=T^{*}\left(\mathbb{R}^{n}\right)$ possesses just $n \in \mathbb{Z}_{+}$ functionally independent invariants in involution, that is a Lie algebra $\mathcal{G}$ is abelian, then it is integrable by quadratures.

In the autonomous case when a Hamiltonian $H=H_{1}$, and invariants $H_{j}: M^{2 n} \rightarrow$ $\mathbb{R}, j=\overline{1, n}$, are independent of the evolution parameter $t \in \mathbb{R}$, the involutivity condition $\left\{H_{i}, H_{j}\right\}=0, i, j=\overline{1, n}$, can be replaced by the weaker one $\left\{H, H_{j}\right\}=c_{j} H$ for some constants $c_{j} \in \mathbb{R}, j=\overline{1, n}$.

The first proof of Theorem 1 was based on a result of S. Lie, which can be formulated as follows.

Theorem 2 (S. Lie). Let vector fields $K_{j} \in \Gamma\left(M^{2 n}\right), j=\overline{1, n}$, be independent in some open neighborhood $U_{h} \in M^{2 n}$, generate a solvable Lie algebra $\mathcal{G}$ with respect to the usual commutator $[\cdot, \cdot]$ on $\Gamma\left(M^{2 n}\right)$ and $\left[K_{j}, K\right]=c_{j} K$ for all $j=\overline{1, n}$, where $c_{j} \in \mathbb{R}, j=\overline{1, n}$, are constants. Then the dynamical system

$$
d u / d t=K(u)
$$

where $u \in U_{h} \subset M^{2 n}$, is integrable by quadratures. 
Example 1 (Motion of three particles on $\mathbb{R}$ under a uniform potential field). The motion of three particles on the axis $\mathbb{R}$ pairwise interacting via a uniform potential field $Q(\|\cdot\|)$ is described as a Hamiltonian system on the canonically symplectic phase space $M=T^{*}\left(\mathbb{R}^{3}\right)$ with the following Lie algebra $\mathcal{G}$ of invariants on $M^{2 n}$ :

$$
\begin{aligned}
H=H_{1} & =\sum_{j=1}^{3} p_{j}^{2} / 2 m_{j}+\sum_{i<j=1}^{3} Q\left(\left\|q_{i}-q_{j}\right\|\right), \\
H_{2} & =\sum_{j=1}^{3} q_{j} p_{j}, \quad H_{3}=\sum_{j=1}^{3} p_{j},
\end{aligned}
$$

where $\left(q_{j}, p_{j}\right) \in T^{*}(\mathbb{R}), j=\overline{1,3}$, are coordinates and momenta of particles on the axis $\mathbb{R}$. The commutation relations for the Lie algebra $\mathcal{G}$ are

$$
\left\{H_{1}, H_{3}\right\}=0, \quad\left\{H_{2}, H_{3}\right\}=H_{3}, \quad\left\{H_{1}, H_{2}\right\}=2 H_{1},
$$

hence it is clearly solvable. Taking a regular element $h \in \mathcal{G}^{*}$, such that $h\left(H_{j}\right)=h_{j}=$ 0 , for $j=1$ and 3 , and $h\left(H_{2}\right)=h_{2} \in \mathbb{R}$ being arbitrary, one obtains the integrability of the problem above in quadratures.

In 1974 V. Arnold proved [4] the following important result known as the commutative (abelian) Liouville-Arnold theorem.

Theorem 3 (J. Liouville - V. Arnold). Suppose a set $\mathcal{G}$ of functions $H_{j}: M^{2 n} \rightarrow \mathbb{R}$, $j=\overline{1, n}$, on a symplectic manifold $M^{2 n}$ is abelian, that is

$$
\left\{H_{i}, H_{j}\right\}=0
$$

for all $i, j=\overline{1, n}$. If on the compact and connected integral submanifold

$$
M_{h}^{n}=\left\{u \in M^{2 n}: h\left(H_{j}\right)=h_{j} \in \mathbb{R}, j=\overline{1, n}, h \in \mathcal{G}^{*}\right\}
$$

with $h \in \mathcal{G}$ being regular, all functions $H: M^{2 n} \rightarrow \mathbb{R}, j=\overline{1, n}$, are functionally independent, then $M_{h}^{n}$ is diffeomorphic to the $n$-dimensional torus $T^{n} \simeq M^{2 n}$, and the motion on it with respect to the Hamiltonian $H=H_{1} \in \mathcal{G}$ is a quasi-periodic function of the evolution parameter $t \in \mathbb{R}$.

A dynamical system satisfying the hypotheses of Theorem 3 is called completely integrable.

In 1978 Mishchenko and Fomenko [2] proved the following generalization of the Liouville-Arnold Theorem 3:

Theorem 4 (A. Mishchenko - A. Fomenko). Assume that on a symplectic manifold $\left(M^{2 n}, \omega^{(2)}\right)$ there is a nonabelian Lie algebra $\mathcal{G}$ of invariants $H_{j}: M \in \mathbb{R}$, $j=\overline{1, k}$, with respect to the dual Poisson bracket on $M^{2 n}$, that is

$$
\left\{H_{i}, H_{j}\right\}=\sum_{s=1}^{k} c_{i j}^{s} H_{s},
$$


where all values $c_{i j}^{s} \in \mathbb{R}, i, j, s=\overline{1, k}$, are constants, and the following conditions are satisfied:

(i) The integral submanifold $M_{h}^{r}:=\left\{u \in M^{2 n}: h\left(H_{j}\right)=h \in \mathcal{G}^{*}\right\}$ is compact and connected at a regular element $h \in \mathcal{G}^{*}$;

(ii) All the functions $H_{j}: M^{2 n} \rightarrow \mathbb{R} j=\overline{1, k}$, are functionally independent on $M^{2 n}$;

(iii) The Lie algebra $\mathcal{G}$ of invariants satisfies the following relationship:

$$
\operatorname{dim} \mathcal{G}+\operatorname{rank} \mathcal{G}=\operatorname{dim} M^{2 n},
$$

where $\operatorname{rank} \mathcal{G}=\operatorname{rank} \mathcal{G}_{h}$ is the dimension of a Cartan subalgebra $\mathcal{G}_{h} \subset \mathcal{G}$.

Then the submanifold $M_{h}^{r} \subset M^{2 n}$ is $r=\operatorname{rank} \mathcal{G}$-dimensional, invariant with respect to each vector field $K \in \Gamma\left(M^{2 n}\right)$, generated by an element $H \in \mathcal{G}_{h}$, and diffeomorphic to the r-dimensional torus $\mathbb{T}^{r} \simeq M_{h}^{r}$, on which the motion is a quasiperiodic function of the evolution parameter $t \in \mathbb{R}$.

The simplest proof of the Mishchenko-Fomenko Theorem 4 can be obtained from the well-known $[3,16]$ classical Lie-Cartan theorem.

Theorem 5 (S. Lie - E. Cartan). Suppose that a point $h \in \mathcal{G}^{*}$ for a given Lie algebra $\mathcal{G}$ of invariants $H_{j}: M^{2 n} \rightarrow \mathbb{R}, j=\overline{1, k}$, is not critical, and the rank $\left\|\left\{H_{i}, H_{j}\right\}: i, j=\overline{1, k}\right\|=2(n-r)$ is constant in an open neighborhood $U_{h} \in \mathbb{R}^{n}$ of the point $\left\{h\left(H_{j}\right)=h_{j} \in \mathbb{R}: j=\overline{1, k}\right\} \subset \mathbb{R}^{k}$. Then in the neighborhood $(h \circ H)^{-1}$ : $U_{h} \subset M^{2 n}$ there exist $k \in \mathbb{Z}_{+}$independent functions $f_{s}: \mathcal{G} \rightarrow \mathbb{R}, s=\overline{1, k}$, such that the functions $F_{s}:=\left(f_{s} \circ H\right): M^{2 n} \in \mathbb{R}, s=\overline{1, k}$, satisfy the following relationships:

$$
\left\{F_{1}, F_{2}\right\}=\left\{F_{3}, F_{4}\right\}=\ldots=\left\{F_{2(n-r)-1}, F_{2(n-r)}\right\}=1,
$$

with all other brackets $\left\{F_{i}, F_{j}\right\}=0$, where $(i, j) \neq(2 s-1,2 s), s=\overline{1, n-r}$. In particular, $(k+r-n) \in \mathbb{Z}_{+}$functions $F_{j}: M^{2 n} \rightarrow \mathbb{R}, j=\overline{1, n-r}$, and $F_{s}: M^{2 n} \rightarrow$ $\mathbb{R}, s=\overline{1, k-2(n-r)}$, compose an abelian algebra $\mathcal{G}_{\tau}$ of new invariants on $M^{2 n}$, independent on $(h \circ H)^{-1}\left(U_{h}\right) \subset M^{2 n}$.

As a simple corollary of the Lie-Cartan Theorem 5 one obtains the following: in the case of the Mishchenko-Fomenko Theorem where $\operatorname{rank} \mathcal{G}+\operatorname{dim} \mathcal{G}=\operatorname{dim} M^{2 n}$, that is $r+k=2 n$, the abelian algebra $\mathcal{G}_{\tau}$ (it is not a subalgebra of $\mathcal{G}$ !) of invariants on $M^{2 n}$ is just $n=1 / 2 \operatorname{dim} M^{2 n}$-dimensional, giving rise to its local complete integrability in $(h \circ H)^{-1}\left(U_{h}\right) \subset M^{2 n}$ via the abelian Liouville-Arnold Theorem 3. It is also evident that the Mishchenko-Fomenko nonabelian integrability Theorem 4 reduces to the commutative (abelian) Liouville-Arnold case when a Lie algebra $\mathcal{G}$ of invariants is just abelian, since then $\operatorname{rank} \mathcal{G}=\operatorname{dim} \mathcal{G}=1 / 2 \operatorname{dim} M^{2 n}=n \in \mathbf{Z}_{+}$, the standard complete integrability condition. 
All the cases of integrability by quadratures described above pose the following fundamental question: How can one effectively construct by means of algebraicanalytical methods the corresponding integral submanifold imbedding

$$
\pi_{h}: M_{h}^{r} \rightarrow M^{2 n},
$$

where $r=\operatorname{rank} \mathcal{G}$, thereby making it possible to express the solutions of an integrable flow on $M_{h}^{r}$ as some exact quasi-periodic functions on the torus $\mathbb{T}^{r} \simeq M_{h}^{r}$.

Below we shall describe an algebraic-analytical algorithm for resolving this question for the case when a symplectic manifold $M^{2 n}$ is diffeomorphic to the canonically symplectic cotangent phase space $T^{*}(\mathbb{R}) \simeq M^{2 n}$.

\section{General Setting}

Our main object of study will be differential systems of vector fields on the cotangent phase space $M^{2 n}=T^{*}\left(\mathbb{R}^{n}\right), n \in \mathbb{Z}_{+}$, endowed with the canonical symplectic structure $\omega^{(2)} \in \Lambda^{2}\left(M^{2 n}\right)$, where by $\omega^{(2)}=d\left(\operatorname{pr}^{*} \alpha^{(1)}\right)$, and

$$
\alpha^{(1)}:=\langle p, d q\rangle=\sum_{j=1}^{n} p_{j} d q_{j},
$$

is the canonical 1-form on the base space $\mathbb{R}^{n}$, lifted naturally to the space $\Lambda^{1}\left(M^{2 n}\right)$, $(q, p) \in M^{2 n}$ are canonical coordinates on $T^{*}\left(\mathbb{R}^{n}\right)$, pr: $T^{*}\left(\mathbb{R}^{n}\right) \rightarrow \mathbb{R}$ is the canonical projection, and $\langle\cdot, \cdot\rangle$ is the usual scalar product in $\mathbb{R}^{n}$.

Assume further that there is also given a Lie subgroup $G$ (not necessarily compact), acting symplectically via the mapping $\varphi: G \times M^{2 n} \rightarrow M^{2 n}$ on $M^{2 n}$, generating a Lie algebra homomorphism $\varphi_{*}: T(\mathcal{G}) \rightarrow \Gamma\left(M^{2 n}\right)$ via the diagram

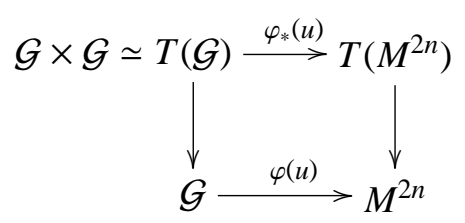

where $u \in M^{2 n}$. Thus, for any $a \in \mathcal{G}$ one can define a vector field $K_{a} \in \Gamma\left(M^{2 n}\right)$ as follows:

$$
K_{a}=\varphi_{*} \cdot a .
$$

Since the manifold $M^{2 n}$ is symplectic, one can naturally define for any $a \in \mathcal{G}$ a function $H_{a} \in \mathcal{D}\left(M^{2 n}\right)$ as follows:

$$
-i_{K_{a}} \omega^{(2)}=d H_{a},
$$

whose existence follows from the invariance property

$$
L_{K_{a}} \omega^{(2)}=0
$$

for all $a \in \mathcal{G}$. The following lemma [1] is useful in applications. 
Lemma 1. If the first homology group $H_{1}(\mathcal{G} ; \mathbb{R})$ of the Lie algebra $\mathcal{G}$ vanishes, then the mapping $\Phi: \mathcal{G} \rightarrow \mathcal{D}\left(M^{2 n}\right)$ defined as

$$
\Phi(a):=H_{a}
$$

for any $a \in \mathcal{G}$ is a Lie algebra homomorphism of $\mathcal{G}$ and $\mathcal{D}\left(M^{2 n}\right)$ (endowed with the Lie structure induced by the symplectic structure $\omega^{(2)} \in \Lambda^{2}\left(M^{2 n}\right)$ ). In this case $\mathcal{G}$ is said to be Poissonian.

As the mapping $\Phi: \mathcal{G} \rightarrow \mathcal{D}\left(M^{2 n}\right)$ is evidently linear in $\mathcal{G}$, expression (2.6) naturally defines a momentum mapping $l: M^{2 n} \rightarrow \mathcal{G}^{*}$ as follows: for any $u \in M^{2 n}$ and all $a \in \mathcal{G}$

$$
(l(u), a)_{\mathcal{G}}:=H_{a}(u),
$$

where $(\cdot, \cdot)_{\mathcal{G}}$ is the standard scalar product on the dual pair $\mathcal{G}^{*} \times \mathcal{G}$. The following characteristic equivariance [1] lemma holds.

Lemma 2. The diagram

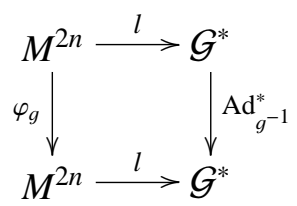

commutes for all $g \in G$, where $\operatorname{Ad}_{g^{-1}}^{*}: \mathcal{G}^{*} \rightarrow \mathcal{G}^{*}$ is the corresponding co-adjoint action of the Lie group $G$ on the dual space $\mathcal{G}^{*}$.

Take now any vector $h \in \mathcal{G}^{*}$ and consider a subspace $\mathcal{G}_{h} \subset \mathcal{G}$, consisting of elements $a \in \mathcal{G}$, such that $\operatorname{ad}_{a}^{*} h=0$, where $\operatorname{ad}_{a}^{*}: \mathcal{G}^{*} \rightarrow \mathcal{G}^{*}$ is the corresponding Lie algebra $\mathcal{G}$ representation in the dual space $\mathcal{G}^{*}$.

The following lemmas hold.

Lemma 3. The subspace $\mathcal{G}_{h} \subset \mathcal{G}$ is a Lie subalgebra of $\mathcal{G}$, called here a Cartan subalgebra.

Lemma 4. Assume a vector $h \in \mathcal{G}^{*}$ is chosen in such a way that $r=\operatorname{dim} \mathcal{G}_{h}$ is minimal. Then the Cartan Lie subalgebra $\mathcal{G}_{h} \subset \mathcal{G}$ is abelian.

In Lemma 4 the corresponding element $h \in \mathcal{G}^{*}$ is called regular and the number $r=\operatorname{dim} \mathcal{G}_{h}$ is called the $\operatorname{rank} \mathcal{G}$ of the Lie algebra $\mathcal{G}$.

About twenty years ago Mishchenko and Fomenko [2] proved the following important noncommutative (nonabelian) Liouville-Arnold theorem.

Theorem 6. On a symplectic space $\left(M^{2 n}, \omega^{(2)}\right)$ let there be given a set of smooth functions $H_{j} \in \mathcal{D}\left(M^{2 n}\right), j=\overline{1, k}$, whose linear span over $\mathbb{R}$ comprises a Lie algebra $\mathcal{G}$ with respect to the corresponding Poisson bracket on $M^{2 n}$. Suppose also that the set

$$
M_{h}^{2 n-k}:=\left\{u \in M^{2 n}: h\left(H_{j}\right)=h_{j} \in \mathbb{R}, j=\overline{1, k}, h \in \mathcal{G}^{*}\right\}
$$


with $h \in \mathcal{G}^{*}$ regular, is a submanifold of $M^{2 n}$, and on $M_{h}^{2 n-k}$ all the functions $H_{j} \in$ $\mathcal{D}\left(M^{2 n}\right), j=\overline{1, k}$, are functionally independent. Assume also that the Lie algebra $\mathcal{G}$ satisfies the following condition:

$$
\operatorname{dim} \mathcal{G}+\operatorname{rank} \mathcal{G}=\operatorname{dim} M^{2 n} .
$$

Then the submanifold $M_{h}^{r}:=M_{h}^{2 n-k}$ is $\operatorname{rank} \mathcal{G}=r$-dimensional and invariant with respect to each vector field $K_{\bar{a}} \in \Gamma\left(M^{2 n}\right)$ with $\bar{a} \in \mathcal{G}_{h} \subset \mathcal{G}$. Given a vector field $K=K_{\bar{a}} \in \Gamma\left(M^{2 n}\right)$ with $\bar{a} \in \mathcal{G}_{h}$ or $K \in \Gamma\left(M^{2 n}\right)$ such that $\left[K, K_{a}\right]=0$ for all $a \in \mathcal{G}$, then, if the submanifold $M_{h}^{r}$ is connected and compact, it is diffeomorphic to the $r$-dimensional torus $T^{r} \simeq M_{h}^{r}$ and the motion of the vector field $K \in \Gamma\left(M^{2 n}\right)$ on it is a quasiperiodic function of the evolution parameter $t \in \mathbb{R}$.

The easiest proof of this result can be obtained from the well-known [3] classical Lie-Cartan theorem, mentioned in the Introduction. Below we shall only sketch the original Mishchenko-Fomenko proof which is heavily based on symplectic theory techniques, some of which have been discussed above.

SkEtch of PRoof. Define a Lie group $G$ naturally as $G=\exp \mathcal{G}$, where $\mathcal{G}$ is the Lie algebra of functions $H_{j} \in \mathcal{D}\left(M^{2 n}\right), j=\overline{1, k}$, in the theorem, with respect to the Poisson bracket $\{\cdot, \cdot\}$ on $M^{2 n}$. Then for an element $h \in \mathcal{G}^{*}$ and any $a=\sum_{j=1}^{k} c_{j} H_{j} \in \mathcal{G}$, where $c_{j} \in \mathbb{R}, j=\overline{1, k}$, the following equality

$$
(h, a)_{\mathcal{G}}:=\sum_{j=1}^{k} c_{j} h\left(H_{j}\right)=\sum_{j=1}^{k} c_{j} h_{j}
$$

holds. Since all functions $H_{j} \in \mathcal{D}\left(M^{2 n}\right), j=\overline{1, k}$, are independent on the level submanifold $M_{h}^{r} \subset M^{2 n}$, this evidently means that the element $h \in \mathcal{G}^{*}$ is regular for the Lie algebra $\mathcal{G}$. Consequently, the Cartan Lie subalgebra $\mathcal{G}_{h} \subset \mathcal{G}$ is abelian. The latter is proved by means of simple straightforward calculations. Moreover, the corresponding momentum mapping $l: M^{2 n} \rightarrow \mathcal{G}^{*}$ is constant on $M_{h}^{r}$ and satisfies the following relation:

$$
l\left(M_{h}^{r}\right)=h \in \mathcal{G}^{*} .
$$

From this it can be shown that all vector fields $K_{\bar{a}} \in \Gamma\left(M^{2 n}\right), \bar{a} \in \mathcal{G}_{h}$, are tangent to the submanifold $M_{h}^{r} \subset M^{2 n}$. Thus the corresponding Lie subgroup $G_{h}:=\exp \mathcal{G}_{h}$ acts naturally and invariantly on $M_{h}^{r}$. If the submanifold $M_{h}^{r} \subset M^{2 n}$ is connected and compact, it follows from (2.9) that $\operatorname{dim} M_{h}^{r}=\operatorname{dim} M^{2 n}-\operatorname{dim} \mathcal{G}=\operatorname{rank} \mathcal{G}=r$, and one obtains via the Arnold theorem [4] that $M_{h}^{r} \simeq \mathbb{T}^{r}$ and the motion of the vector field $K \in \Gamma\left(M^{2 n}\right)$ is a quasiperiodic function of the evolution parameter $t \in \mathbb{R}$, thus proving the theorem. 
As a nontrivial consequence of the Lie-Cartan theorem mentioned before and of Theorem 6, one can prove the following dual theorem about abelian Liouville-Arnold integrability.

Theorem 7. Let a vector field $K \in \Gamma\left(M^{2 n}\right)$ be completely integrable via the nonabelian scheme of Theorem 6. Then it is also Liouville-Arnold integrable on $M^{2 n}$ and possesses, under some additional conditions, yet another abelian Lie algebra $\mathcal{G}_{h}$ of functionally independent invariants on $M^{2 n}$, for which $\operatorname{dim} \mathcal{G}_{h}=n=(1 / 2) \operatorname{dim} M^{2 n}$.

The available proof of the theorem above is quite complicated, and we shall comment on it in detail later on. We mention here only that some analogs of the reduction Theorem 6 for the case where $M^{2 n} \simeq \mathcal{G}^{*}$, so that an arbitrary Lie group $G$ acts symplectically on the manifold, were proved also in $[6-10,34]$. Notice here, that in the case when equality (2.10) is not satisfied one can then construct in the usual way the reduced manifold $\bar{M}_{h}^{2 n-k-r}:=M_{h}^{2 n-k} / G_{h}$ on which there exists a symplectic structure $\bar{\omega}_{h}^{(2)} \in \Lambda^{2}\left(\bar{M}_{h}^{2 n-k-r}\right)$, defined as

$$
r_{h}^{*} \bar{\omega}_{h}^{(2)}=\pi_{h}^{*} \omega^{(2)}
$$

with respect to the following compatible reduction-imbedding diagram:

$$
\bar{M}_{h}^{2 n-k-r} \stackrel{r_{h}}{\longleftarrow} M_{h}^{2 n-k} \stackrel{\pi_{h}}{\longrightarrow} M^{2 n}
$$

where $r_{h}: M_{h}^{2 n-k} \rightarrow \bar{M}_{h}^{2 n-k-r}$ and $\pi_{h}: M_{h}^{2 n-k} \rightarrow M^{2 n}$ are, respectively, the corresponding reductions and imbedding mappings. The nondegeneracy of the 2-form $\bar{\omega}_{h}^{(2)} \in \Lambda^{2}\left(\bar{M}_{h}\right)$ defined by (2.13), follows simply from the expression

$$
\begin{aligned}
\operatorname{ker}\left(\pi_{h}^{*} \omega^{(2)}(u)\right) & =T_{u}\left(M_{h}^{2 n-k}\right) \cap T_{u}^{\perp}\left(M_{h}^{2 n-k}\right) \\
& =\operatorname{span}_{\mathbb{R}}\left\{K_{\bar{a}}(u) \in T_{u}\left(\bar{M}_{h}^{2 n-k-r}:=M_{h}^{2 n-k} / G_{h}\right): \bar{a} \in \mathcal{G}_{h}\right\}
\end{aligned}
$$

for any $u \in M_{h}^{2 n-k}$, since all vector fields $K_{\bar{a}} \in \Gamma\left(M^{2 n}\right), \bar{a} \in \mathcal{G}_{h}$, are tangent to $\bar{M}_{h}^{2 n-k-r}:=M_{h}^{2 n-k} / G_{h}$. Thus, the reduced space $\bar{M}_{h}^{2 n-k-r}:=M_{h}^{2 n-k} / G_{h}$ with respect to the orbits of the Lie subgroup $G_{h}$ action on $M_{h}^{2 n-k}$ will be a $(2 n-k-r)$-dimensional symplectic manifold. The latter evidently means that the number $2 n-k-r=2 s \in \mathbb{Z}_{+}$ is even as there is no symplectic structure on odd-dimensional manifolds. This obviously is closely connected with the problem of existence of a symplectic group action of a Lie group $G$ on a given symplectic manifold $\left(M^{2 n}, \omega^{(2)}\right)$ with a symplectic structure $\omega^{(2)} \in \Lambda^{(2)}\left(M^{2 n}\right)$ being a priori fixed. From this point of view one can consider the inverse problem of constructing symplectic structures on a manifold $M^{2 n}$, admitting a Lie group $G$ action. Namely, owing to the equivariance property (2.8) of the momentum mapping $l: M^{2 n} \rightarrow \mathcal{G}^{*}$, one can obtain the induced symplectic structure $l^{*} \Omega_{h}^{(2)} \in \Lambda^{2}\left(\bar{M}_{h}^{2 n-k-r}\right)$ on $\bar{M}_{h}^{2 n-k-r}$ from the canonical symplectic structure $\Omega_{h}^{(2)} \in \Lambda^{(2)}(\operatorname{Or}(h ; G))$ on the orbit $\operatorname{Or}(h ; G) \subset \mathcal{G}^{*}$ of a regular element $h \in \mathcal{G}^{*}$. 
Since the symplectic structure $l^{*} \Omega_{h}^{(2)} \in \Lambda^{2}\left(\bar{M}_{h}\right)$ can be naturally lifted to the 2-form $\tilde{\omega}^{(2)}=\left(r_{h}^{*} \circ l^{*}\right) \Omega_{h}^{(2)} \in \Lambda^{2}\left(M_{h}^{2 n-k}\right)$, the latter being degenerate on $M_{h}^{2 n-k}$ can apparently be nonuniquely extended on the whole manifold $M^{2 n}$ to a symplectic structure $\omega^{(2)} \in \Lambda^{2}\left(M^{2 n}\right)$, for which the action of the Lie group $G$ is a priori symplectic. Thus, many properties of a given dynamical system with a Lie algebra $\mathcal{G}$ of invariants on $M^{2 n}$ are deeply connected with the symplectic structure $\omega^{(2)} \in \Lambda^{2}\left(M^{2 n}\right)$ the manifold $M^{2 n}$ is endowed with, and in particular, with the corresponding integral submanifold imbedding mapping $\pi_{h}: M_{h}^{2 n-k} \rightarrow M^{2 n}$ at a regular element $h \in \mathcal{G}^{*}$. The problem of direct algebraic-analytical construction of this mapping was in part solved in [11] in the case where $n=2$ for an abelian algebra $\mathcal{G}$ on the manifold $M^{4}=T^{*}\left(\mathbb{R}^{2}\right)$. The treatment of this problem in [11] was extensively based both on the classical Cartan studies of integral submanifolds of ideals in Grassmann algebras and on the modern Galisot-Reeb-Françoise results for a symplectic manifold $\left(M^{2 n}, \omega^{(2)}\right)$ structure, on which there exists an involutive set $\mathcal{G}$ of functionally independent invariants $H_{j} \in \mathcal{D}\left(M^{2 n}\right), j=\overline{1, n}$. In what follows below we generalize the Galisot-Reeb-Françoise results to the case of a nonabelian set of functionally independent functions $H_{j} \in \mathcal{D},\left(M^{2 n}\right), j=\overline{1, k}$, comprising a Lie algebra $\mathcal{G}$ and satisfying the Mishchenko-Fomenko condition (2.9): $\operatorname{dim} \mathcal{G}+\operatorname{rank} \mathcal{G}=\operatorname{dim} M^{2 n}$. This makes it possible to devise an effective algebraic-analytical method of constructing the corresponding integral submanifold imbedding and reduction mappings, giving rise to a wide class of exact, integrable by quadratures, solutions of a given integrable vector field on $M^{2 n}$.

\section{INTEGRAL SUBMANIFOLD IMBEDDING PROBLEM FOR AN ABELIAN LIE ALGEBRA OF INVARIANTS}

We shall consider here only a set $\mathcal{G}$ of commuting polynomial functions $H_{j} \in$ $\mathcal{D}\left(M^{2 n}\right), j=\overline{1, n}$, on the canonically symplectic phase space $M^{2 n}=T^{*}\left(\mathbb{R}^{n}\right)$. Due to the Liouville-Arnold theorem [4], any dynamical system $K \in \Gamma\left(M^{2 n}\right)$ commuting with corresponding Hamiltonian vector fields $K_{a}$ for all $a \in \mathcal{G}$, will be integrable by quadratures in case of a regular element $h \in \mathcal{G}^{*}$, which defines the corresponding integral submanifold $M_{h}^{n}:=\left\{u \in M^{2 n}: h\left(H_{j}\right)=h_{j} \in \mathbb{R}, j=\overline{1, n}\right\}$ which is diffeomorphic (when compact and connected) to the $n$-dimensional torus $\mathbb{T}^{n} \simeq M_{h}^{n}$. This in particular means that there exists some algebraic-analytical expression for the integral submanifold imbedding mapping $\pi_{h}: M_{h}^{n} \rightarrow M^{2 n}$ into the ambient phase space $M^{2 n}$, which one should find in order to properly demonstrate integrability by quadratures.

The problem formulated above was posed and in part solved (as was mentioned above) for $n=2$ in [11] and in [13] for a Henon-Heiles dynamical system which had previously been integrated $[14,15]$ using other tools. Here we generalize the approach of [11] for the general case $n \in \mathbb{Z}_{+}$and proceed further in Section 3 to solve 
this problem in the case of a nonabelian Lie algebra $\mathcal{G}$ of polynomial invariants on $M^{2 n}=T^{*}\left(\mathbb{R}^{n}\right)$, satisfying all the conditions of the Mishchenko-Fomenko Theorem 6.

Define now the basic vector fields $K_{j} \in \Gamma\left(M^{2 n}\right), j=\overline{1, n}$, generated by basic elements $H_{j} \in \mathcal{G}$ of an abelian Lie algebra $\mathcal{G}$ of invariants on $M^{2 n}$, as follows:

$$
-i_{K_{j}} \omega^{(2)}=d H_{j}
$$

for all $j=\overline{1, n}$. It is easy to see that the condition $\left\{H_{j}, H_{i}\right\}=0$ for all $i, j=\overline{1, n}$, yields also $\left[K_{i}, K_{j}\right]=0$ for all $i, j=\overline{1, n}$. Taking into account that $\operatorname{dim} M^{2 n}=2 n$, one obtains the equality $\left(\omega^{(2)}\right)^{n}=0$ identically on $M^{2 n}$. This makes it possible to formulate the following Galisau-Reeb result.

Theorem 8. Assume that an element $h \in \mathcal{G}^{*}$ is chosen to be regular and a Lie algebra $\mathcal{G}$ of invariants on $M^{2 n}$ is abelian. Then there exist differential 1-forms $h_{j}^{(1)} \in \Lambda^{1}\left(U\left(M_{h}^{n}\right)\right), j=\overline{1, n}$, where $U\left(M_{h}^{n}\right)$ is some open neighborhood of the integral submanifold $M_{h}^{n} \subset M^{2 n}$, satisfying the following properties:

(i) $\left.\omega^{(2)}\right|_{U\left(M_{h}^{n}\right)}=\sum_{j=1}^{n} d H_{j} \wedge h_{j}^{(1)}$;

(ii) The exterior differentials $d h_{j}^{(1)} \in \Lambda^{2}\left(U\left(M_{h}^{n}\right)\right)$ belong to the ideal $\mathcal{I}(\mathcal{G})$ in the Grassmann algebra $\Lambda\left(U\left(M_{h}^{n}\right)\right)$ generated by the 1-forms $d H_{j} \in \Lambda^{1}\left(U\left(M_{h}^{n}\right)\right)$, $j=\overline{1, n}$.

Proof. Consider the following identity on $M^{2 n}$ :

$$
\left(\otimes_{j=1}^{n} i_{K_{j}}\right)\left(\omega^{(2)}\right)^{n+1}=0= \pm(n+1) !\left(\wedge_{j=1}^{n} d H_{j}\right) \wedge \omega^{(2)},
$$

which implies that the 2-form $\omega^{(2)} \in \mathcal{I}(\mathcal{G})$. Therefore, one can find 1-forms $h_{j}^{(1)} \in$ $\Lambda^{1}\left(U\left(M_{h}^{n}\right)\right), j=\overline{1, n}$, satisfying the condition

$$
\left.\omega^{(2)}\right|_{U\left(M_{h}^{n}\right)}=\sum_{j=1}^{n} d H_{j} \wedge h_{j}^{(1)} .
$$

Since $\omega^{(2)} \in \Lambda^{2}\left(U\left(M_{h}^{n}\right)\right)$ is nondegenerate on $M^{2 n}$, it follows that all 1-forms $h_{j}^{(1)}$, $j=\overline{1, n}$, in (3.3) are independent on $U\left(M_{h}^{n}\right)$, proving part (i) of the Theorem. As $d \omega^{(2)}=0$ on $M^{2 n}$, from (2.3) one gets that

$$
\sum_{j=1}^{n} d H_{j} \wedge d h_{j}^{(1)}=0
$$

on $U\left(M_{h}^{n}\right)$, hence it is obvious that $d h_{j}^{(1)} \in \mathcal{I}(\mathcal{G}) \subset \Lambda\left(U\left(M_{h}^{n}\right)\right)$ for all $j=\overline{1, n}$, proving part (ii) of the Theorem. 
Now we proceed to study properties of the integral submanifold $M_{h}^{n} \subset M^{2 n}$ of the ideal $\mathcal{I}(\mathcal{G})$ in the Grassmann algebra $\Lambda\left(U\left(M_{h}^{n}\right)\right.$ ). In general, the integral submanifold $M_{h}^{n}$ is completely described [16] by means of the imbedding

$$
\pi_{h}: M_{h}^{n} \rightarrow M^{2 n}
$$

and using this, one can reduce all vector fields $K_{j} \in \Gamma\left(M^{2 n}\right), j=\overline{1, n}$, on the submanifold $M_{h}^{n} \subset M^{2 n}$, since they are all evidently in its tangent space. If $\bar{K}_{j} \in \Gamma\left(M_{h}^{n}\right)$, $j=\overline{1, n}$, are the corresponding pulled-back vector fields $K_{j} \in \Gamma\left(M^{2 n}\right), j=\overline{1, n}$, then by definition, the equality

$$
\pi_{h *} \circ \bar{K}_{j}=K_{j} \circ \pi_{h}
$$

holds for all $j=\overline{1, n}$. Similarly one can construct 1 -forms $\bar{h}_{j}^{(1)}:=\pi_{h}^{*} \circ h_{j}^{(1)} \in \Lambda^{1}\left(M_{h}^{n}\right)$, $j=\overline{1, n}$, which are characterized by the following Cartan-Jost [16] Theorem.

Theorem 9. The following assertions are true:

(i) The 1-forms $\bar{h}_{j}^{(1)} \in \Lambda^{1}\left(M_{h}^{n}\right), j=\overline{1, n}$, are independent on $M_{h}^{n}$;

(ii) The 1 -forms $\bar{h}_{j}^{(1)} \in \Lambda^{1}\left(M_{h}^{n}\right), j=\overline{1, n}$, are exact on $M_{h}^{n}$ and satisfy $\bar{h}_{j}^{(1)}\left(\bar{K}_{j}\right)=$ $\delta_{i j}, i, j=\overline{1, n}$.

Proof. As the ideal $\mathcal{I}(\mathcal{G})$ is by definition vanishing on $M_{h}^{n} \subset M^{2 n}$ and closed on $U\left(M_{h}^{n}\right)$, the integral submanifold $M_{h}^{n}$ is well defined in the case of a regular element $h \in \mathcal{G}^{*}$. This implies that the imbedding (3.5) is nondegenerate on $M_{h}^{n} \subset M^{2 n}$, or the 1 -forms $\bar{h}_{j}^{(1)}:=\pi_{h}^{*} \circ h_{j}^{(1)}, j=\overline{1, n}$, will persist in being independent if they are 1 -forms $h_{j}^{(1)} \in \Lambda^{1}\left(U\left(M_{h}^{n}\right)\right), j=\overline{1, n}$, proving part (i) of the theorem. Using property (ii) of Theorem 8, one sees that on the integral submanifold $M_{h}^{n} \subset M^{2 n}$ all 2-forms $d \bar{h}_{j}^{(1)}=0, j=\overline{1, n}$. Consequently, owing to the Poincaré lemma $[1,16]$, the 1-forms $\bar{h}_{j}^{(1)}=d \bar{t}_{j} \in \Lambda^{1}\left(M_{h}^{n}\right), j=\overline{1, n}$, for some mappings $\bar{t}_{j}: M_{h}^{n} \rightarrow \mathbb{R}, j=\overline{1, n}$, defining global coordinates on an appropriate universal covering of $M_{h}^{n}$. Consider now the following identity based on the representation (3.3):

$$
\left.i_{K_{j}} \omega^{(2)}\right|_{U\left(M_{h}^{n}\right)}=-\sum_{i=1}^{n} h_{i}^{(1)}\left(K_{j}\right) d H_{i}:=-d H_{j}
$$

which holds for any $j=\overline{1, n}$. As all $d H_{j} \in \Lambda^{1}\left(U\left(M_{h}^{n}\right)\right), j=\overline{1, n}$, are independent, from (3.7) one infers that $h_{i}^{(1)}\left(K_{j}\right)=\delta_{i j}$ for all $i, j=\frac{h}{1, n}$. Recalling now that for any $i=\overline{1, n}, K_{i} \circ \pi_{h}=\pi_{h *} \circ K_{i}$, one readily computes that

$$
\bar{h}_{i}^{(1)}\left(\bar{K}_{j}\right)=\pi_{h}^{*} h_{i}^{(1)}\left(\bar{K}_{j}\right):=h_{i}^{(1)}\left(\pi_{h *} \circ K_{j}\right):=h_{i}^{(1)}\left(K_{j} \circ \pi_{h}\right)=\delta_{i j}
$$

for all $i, j=\overline{1, n}$, proving part (ii) of the Theorem.

The following is a simple consequence of Theorem 9: 
Corollary 2. Suppose that the vector fields $K_{j} \in \Gamma\left(M^{2 n}\right), j=\overline{1, n}$, are parametrized globally along their trajectories by means of the corresponding parameters $t_{j}$ : $M^{2 n} \rightarrow \mathbb{R}, j=\overline{1, n}$, that is on the phase space $M^{2 n}$

$$
d / d t_{j}:=K_{j}
$$

for all $j=\overline{1, n}$. Then the following important equalities hold (up to constant normalizations) on the integral submanifold $M_{h}^{n} \subset M^{2 n}$ :

$$
\left.t_{j}\right|_{M_{h}^{n}}=\bar{t}_{j},
$$

where $1 \leq j \leq n$.

We consider a completely integrable via Liouville-Arnold Hamiltonian system on the cotangent canonically symplectic manifold $\left(T^{*}\left(\mathbb{R}^{n}\right), \omega^{(2)}\right), n \in \mathbb{Z}_{+}$, possessing exactly $n \in \mathbb{Z}_{+}$functionally independent and Poisson commuting algebraic polynomial invariants $H_{j}: T^{*}\left(\mathbb{R}^{n}\right) \rightarrow \mathbb{R}, j=\overline{1, n}$. Due to the Liouville-Arnold theorem this Hamiltonian system can be completely integrated by quadratures in quasiperiodic functions on its integral submanifold when taken compact. It is equivalent to the statement that this compact integral submanifold is diffeomorphic to a torus $\mathbb{T}^{n}$, which makes it possible to formulate the problem of integrating the system by means of searching the corresponding integral submanifold imbedding mapping $\pi_{h}: M_{h}^{n} \longrightarrow T^{*}\left(\mathbb{R}^{n}\right)$, where by definition

$$
M_{h}^{n}:=\left\{(q, p) \in T^{*}\left(\mathbb{R}^{n}\right): H_{j}(q, p)=h_{j} \in \mathbb{R}, j=\overline{1, n}\right\} .
$$

Since $M_{h}^{n} \simeq \mathbb{T}^{n}$, and the integral submanifold (1.1) is invariant subject to all Hamiltonian flows $K_{j}: T^{*}\left(\mathbb{R}^{n}\right) \rightarrow T\left(T^{*}\left(\mathbb{R}^{n}\right)\right), j=\overline{1, n}$, where

$$
i_{K_{j}} \omega^{(2)}=-d H_{j},
$$

there exist corresponding "action-angle" coordinates $(\varphi, \gamma) \in\left(\mathbb{T}_{\gamma}^{n}, \mathbb{R}^{n}\right)$ on the torus $\mathbb{T}_{\gamma}^{n} \simeq M_{h}^{n}$, specifying its imbedding $\pi_{\gamma}: \mathbb{T}_{\gamma}^{n} \rightarrow T^{*}\left(\mathbb{R}^{n}\right)$ by means of a set of smooth functions $\gamma \in \mathcal{D}\left(\mathbb{R}^{n}\right)$, where

$$
\mathbb{T}_{\gamma}^{n}:=\left\{(q, p) \in T^{*}\left(\mathbb{R}^{n}\right): \gamma_{j}(H)=\gamma_{j} \in \mathbb{R}, j=\overline{1, n}\right\} .
$$

The induced by (3.12) mapping $\gamma: \mathbb{R}^{n} \ni h \rightarrow \mathbb{R}^{n}$ is of great interest for many applications and was studied still earlier by Picard and Fuchs subject to the corresponding differential equations it satisfies:

$$
\partial \gamma_{j}(h) / \partial h_{i}=F_{i j}(\gamma ; h),
$$

where $h \in \mathbb{R}^{n}$ and $F_{i j}: \mathbb{R}^{n} \times \mathbb{R}^{n} \rightarrow \mathbb{R}, i, j=\overline{1, n}$, are some almost everywhere smooth functions. In the case when the right hand side of (1.5) is a set of algebraic functions on $\mathbb{C}^{n} \times \mathbb{C}^{n} \ni(\gamma ; h)$, all Hamiltonian flows $K_{j}: T^{*}\left(\mathbb{R}^{n}\right) \rightarrow T\left(T^{*}\left(\mathbb{R}^{n}\right)\right)$, $j=\overline{1, n}$, are said to be algebraically completely integrable in quadratures. In general equations like (3.13) were studied in [19,31], a recent example can be found in [18]. 
It is clear enough that Picard-Fuchs equations (3.13) are related to the associated canonical transformation of the symplectic 2-form $\omega^{(2)} \in \Lambda^{2}\left(T^{*}\left(\mathbb{R}^{n}\right)\right)$ in a neighborhood $U\left(M_{h}^{n}\right)$ of the integral submanifold $M_{h}^{n} \subset T^{*}\left(\mathbb{R}^{n}\right)$. To make it more precise, denote $\omega^{(2)}(q, p)=d \operatorname{pr}^{*} \alpha^{(1)}(q ; p)$, where for $(q, p) \in T^{*}\left(\mathbb{R}^{n}\right)$

$$
\alpha^{(1)}(q ; p):=\sum_{j=1}^{n} p_{j} d q_{j}=\langle p, d q\rangle \in \Lambda^{1}\left(\mathbb{R}^{n}\right)
$$

is the canonical Liouville 1 -form on $\mathbb{R}^{n},\langle\cdot, \cdot\rangle$ is the usual scalar product in $\mathbb{R}^{n}$, pr : $T^{*}\left(\mathbb{R}^{n}\right) \rightarrow \mathbb{R}^{n}$ is the bundle projection. One can now define a mapping

$$
d S_{q}: \mathbb{R}^{n} \rightarrow T_{q}^{*}\left(\mathbb{R}^{n}\right)
$$

such that $d S_{q}(h) \in T_{q}^{*}\left(\mathbb{R}^{n}\right)$ is an exact 1-form for all $q \in M_{h}^{n}$ and $h \in \mathbb{R}^{n}$, yielding

$$
\left(d S_{q}\right)^{*}\left(d \operatorname{pr}^{*} \alpha^{(1)}\right)=\left(d S_{q}\right)^{*} \omega^{(2)}:=d^{2} S_{q} \equiv 0 .
$$

Thereby the mapping (3.15) defines a so-called generating function [1,2] $S_{q}: \mathbb{R}^{n} \rightarrow$ $\mathbb{R}$, satisfying on $M_{h}^{n}$ the relationship

$$
\operatorname{pr}^{*} \alpha^{(1)}(q ; p)+\langle t, d h\rangle=d S_{q}(h),
$$

where $t \in \mathbb{R}^{n}$ is the set of evolution parameters. From (3.17) one gets right away that equality

$$
S_{q}(h)=\left.\int_{q^{(0)}}^{q}\langle p, d q\rangle\right|_{M_{h}^{n}}
$$

holds for any $q, q^{(0)} \in M_{h}^{n}$. On the other hand, one can define one more generating function $S_{\mu}: \mathbb{R}^{n} \rightarrow \mathbb{R}$, such that

$$
d S_{\mu}: \mathbb{R}^{n} \rightarrow T_{\mu}^{*}\left(M_{h}^{n}\right)
$$

where $\mu \in M_{h}^{n} \simeq \otimes_{j=1}^{n} \mathbb{S}_{j}^{1}$ are global separable coordinates existing on $M_{h}^{n}$ owing to the Liouville-Arnold theorem. Thus one can write the following canonical relationsip:

$$
\langle w, d \mu\rangle+\langle t, d h\rangle=d S_{\mu}(h),
$$

where $w_{j}:=w_{j}\left(\mu_{j} ; h\right) \in T_{\mu_{j}}^{*}\left(\mathbf{S}_{j}^{1}\right)$ for every $j=\overline{1, n}$. Whence it follows readily that

$$
S_{\mu}(h)=\sum_{j=1}^{n} \int_{\mu_{j}^{(0)}}^{\mu_{j}} w(\lambda ; h) d \lambda,
$$

satisfying on $M_{h}^{n} \subset T^{*}\left(\mathbb{R}^{n}\right)$ the following relationship

$$
d S_{\mu}+d \mathcal{L}_{\mu}=\left.d S_{q}\right|_{q=q(\mu ; h)}
$$

for some mapping $\mathcal{L}_{\mu}: \mathbb{R}^{n} \rightarrow \mathbb{R}$. As a result of (3.21) and (3.22) one gets that the following important expressions

$$
t_{i}=\partial S_{\mu}(h) / \partial h_{i}, \quad\left\langle p, \partial q / \partial \mu_{i}\right\rangle=w_{i}+\partial \mathcal{L}_{\mu} / \partial \mu_{i}
$$


hold for all $i=\overline{1, n}$. A construction similar to the above can be done subject to the imbedded torus $\mathbb{T}_{\gamma}^{n} \subset T^{*}\left(\mathbb{R}^{n}\right)$ :

$$
d \tilde{S}_{q}(\gamma):=\sum_{j=1}^{n} p_{j} d q_{j}+\sum_{i=1}^{n} \varphi_{i} d \gamma_{i}
$$

where owing to (3.15) $\tilde{S}_{q}(\gamma):=S_{q}(\xi \cdot \gamma), \xi \cdot \gamma(h)=h$, for all $(q ; \gamma) \in U\left(M_{h}^{n}\right)$. For angle coordinates $\varphi \in \mathbb{T}_{\gamma}^{n}$ one obtains from (3.24) that

$$
\varphi_{i}=\partial \tilde{S}_{q}(\gamma) / \partial \gamma_{i}
$$

for all $i=\overline{1, n}$. As $\varphi_{i} \in \mathbb{R} / 2 \pi \mathbb{Z}, i=\overline{1, n}$, from (3.26) one derives that

$$
\frac{1}{2 \pi} \oint_{\sigma_{j}^{(h)}} d \varphi_{i}=\delta_{i j}=\frac{1}{2 \pi} \frac{\partial}{\partial \gamma_{i}} \oint_{\sigma_{j}^{(h)}} d \tilde{S}_{q}(\gamma)=\frac{1}{2 \pi} \frac{\partial}{\partial \gamma_{i}} \oint_{\sigma_{j}^{(h)}}\langle p, d q\rangle
$$

for all canonical cycles $\sigma_{j}^{(h)} \subset M_{h}^{n}, j=\overline{1, n}$, constituting a basis of the one dimensional homology group $H^{1}\left(M_{h}^{n} ; \mathbb{Z}\right)$. Thereby, owing to (3.26), it follows that for all $i=\overline{1, n}$ "action" variables can be found as

$$
\gamma_{i}=\frac{1}{2 \pi} \oint_{\sigma_{i}^{(h)}}\langle p, d q\rangle
$$

Recall now that $M_{h}^{n} \simeq \mathbb{T}_{\gamma}^{n}$ are diffeomorphic also to $\otimes_{j=1}^{n} \mathbb{S}_{j}^{1}$, where $\mathbb{S}_{j}^{1}, j=\overline{1, n}$, are some one-dimensional real circles. The evolution along any of the vector fields $K_{j}: T^{*}\left(\mathbb{R}^{n}\right) \rightarrow T\left(T^{*}\left(\mathbb{R}^{n}\right)\right), j=\overline{1, n}$, on $M_{h}^{n} \subset T^{*}\left(\mathbb{R}^{n}\right)$ is known [1,2] to be a linear winding around the torus $\mathbb{T}_{\gamma}^{n}$, that can be interpreted also this way: the above introduced independent of every other global coordinate on circles $\mathbb{S}_{j}^{1}, j=\overline{1, n}$, are such that the resulting evolution undergoes a quasiperiodic motion. These coordinates being still called Hamilton-Jacobi ones prove to be very important for accomplishing the complete integrability by quadratures via solving the corresponding Picard-Fuchs type equations.

Let us denote these separable coordinates on the integral submanifold $M_{h}^{n} \simeq \otimes_{j=1}^{n} \mathbb{S}_{j}^{1}$ by $\mu_{j} \in \mathbb{S}_{j}^{1}, j=\overline{1, n}$, and define the corresponding imbedding mapping $\pi_{h}: M_{h}^{n} \rightarrow$ $T^{*}\left(\mathbb{R}^{n}\right)$ as

$$
q=q(\mu ; h), p=p(\mu ; h) .
$$

There exist two important cases subject to the imbedding (3.28) .

The first case is related to the integral submanifold $M_{h}^{n} \subset T^{*}\left(\mathbb{R}^{n}\right)$ which can be parametrized as a manifold by means of the base coordinates $q \in \mathbb{R}^{n}$ of the cotangent bundle $T^{*}\left(\mathbb{R}^{n}\right)$. This can be explained as follows: the canonical Liouville 1-form $\alpha^{(1)} \in \Lambda^{1}\left(\mathbb{R}^{n}\right)$, in accordance with the diagram 


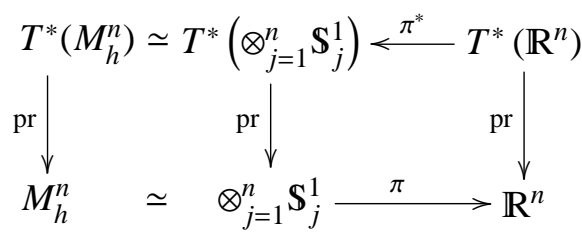

is mapped by the imbedding mapping $\pi=\mathrm{pr} \cdot \pi_{h}: M_{h}^{n} \rightarrow \mathbb{R}^{n}$ not depending on a set of parameters $h \in \mathbb{R}^{n}$, into the 1 -form

$$
\alpha_{h}^{(1)}=\pi^{*} \alpha^{(1)}=\sum_{j=1}^{n} w_{j}\left(\mu_{j} ; h\right) d \mu_{j}
$$

where $(\mu, w) \in T^{*}\left(\otimes_{j=1}^{n} \mathbb{S}_{j}^{1}\right) \simeq \otimes_{j=1}^{n} T^{*}\left(\mathbf{S}_{j}^{1}\right)$. The imbedding mapping $\pi: M_{h}^{n} \rightarrow \mathbb{R}^{n}$ due to equality (3.30) makes the function $\mathcal{L}_{\mu}: \mathbb{R}^{n} \rightarrow \mathbb{R}$ to be zero giving rise to the generating function $S_{\mu}: \mathbb{R}^{n} \rightarrow \mathbb{R}$, enjoying the condition

$$
d S_{\mu}=\left.d S_{q}\right|_{q=q(\mu ; h)}
$$

where as before

$$
S_{\mu}(h)=\sum_{j=1}^{n} p_{j} d q_{j}+\sum_{j=1}^{n} t_{j} d h_{j}
$$

and $\operatorname{det}\|\partial q(\mu ; h) / \partial \mu\| \neq 0$ almost everywhere on $M_{h}^{n}$ for all $h \in \mathbb{R}^{n}$. Similarly to (3.23), one gets from (3.32) that

$$
t_{j}=\partial S_{\mu}(h) / \partial h_{j}
$$

for $j=\overline{1, n}$. Concerning the second part of the imbedding mapping (3.28) we arrive due to equality (3.30) at the following simple result:

$$
p_{i}=\sum_{j=1}^{n} w_{j}\left(\mu_{j} ; h\right) \partial \mu_{j} / \partial q_{i}
$$

where $i=\overline{1, n}$ and $\operatorname{det}\|\partial \mu / \partial q\| \neq 0$ almost everywhere on $\pi\left(M_{h}^{n}\right)$ due to the local invertibility of the imbedding mapping $\pi: M_{h}^{n} \rightarrow \mathbb{R}^{n}$. Thus, we can claim that the problem of complete integrability in the first case is solved iff the only imbedding mapping $\pi: M_{h}^{n} \rightarrow \mathbb{R}^{n} \subset T^{*}\left(\mathbb{R}^{n}\right)$ is constructed. This case was in detail considered in [11], where the corresponding Picard-Fuchs type equations were built based on an extension of Galisot-Reeb and Françoise results [18]. Namely, similarly to (3.13), these equations are defined as follows:

$$
\partial w_{j}\left(\mu_{j} ; h\right) / \partial h_{k}=P_{k j}\left(\mu_{j}, w_{j} ; h\right),
$$

where $P_{k j}: T^{*}\left(\otimes_{j=1}^{n} \mathbb{S}_{j}^{1}\right) \times \mathbb{C}^{n} \rightarrow \mathbb{C}, k, j=\overline{1, n}$, are some algebraic functions of their arguments. 
Concerning the second case when the integral submanifold $M_{h}^{n} \subset T^{*}\left(\mathbb{R}^{n}\right)$ cannot be imbedded almost everywhere into the base space $\mathbb{R}^{n} \subset T^{*}\left(\mathbb{R}^{n}\right)$, the relationship like (3.32) does not take place, and we are forced to consider the usual canonical transformation from $T^{*}\left(\mathbb{R}^{n}\right)$ to $T^{*}\left(\mathbb{R}^{n}\right)$ based on a mapping $d \mathcal{L}_{q}: \otimes_{j=1}^{n} \mathbb{S}_{j}^{1} \rightarrow$ $T^{*}\left(\mathbb{R}^{n}\right)$, where $\mathcal{L}_{q}: \otimes_{j=1}^{n} \mathbb{S}_{j}^{1} \rightarrow \mathbb{R}$ enjoys for all $\mu \in \otimes_{j=1}^{n} \mathbb{S}_{j}^{1} \simeq M_{h}^{n} \ni q$ the following relationship

$$
\operatorname{pr}^{*} \alpha^{(1)}(q ; p)=\sum_{j=1}^{n} w_{j} d \mu_{j}+d L_{q}(\mu) .
$$

In this case we can derive for any $\mu \in \otimes_{j=1}^{n} \mathbb{S}_{j}^{1}$ the previously introduced hereditary generating function $\mathcal{L}_{\mu}: \mathbb{R}^{n} \rightarrow T^{*}\left(\otimes_{j=1}^{n} \mathbb{S}_{j}^{1}\right)$ as

$$
d \mathcal{L}_{\mu}=\left.d \mathcal{L}_{q}\right|_{q=q(\mu ; h)}
$$

satisfying evidently the following canonical transformation condition:

$$
d S_{q}(h)=\sum_{j=1}^{n} w_{j}\left(\mu_{j} ; h\right) d \mu_{j}+\sum_{j=1}^{n} t_{j} d h_{j}+d L_{\mu}(h),
$$

for almost all $\mu \in \otimes_{j=1}^{n} \mathbb{S}_{j}^{1}$ and $h \in \mathbb{R}^{n}$. Based on (3.38) one can derive the following relationships:

$$
\partial L_{\mu}(h) / \partial h_{j}=\left.\left\langle p, \partial q / \partial h_{j}\right\rangle\right|_{M_{h}^{n}}
$$

for all $j=\overline{1,2}, \mu \in \otimes_{j=1}^{n} \mathbb{S}_{j}^{1}$ and $h \in \mathbb{R}^{n}$. Whence the following important analytical result,

$$
\begin{gathered}
t_{s}=\sum_{j=1}^{n} \int_{\mu_{j}^{(0)}}^{\mu_{j}}\left(\partial w_{j}(\lambda ; h) / \partial h_{s}\right) d \lambda, \\
\sum_{j=1}^{n} p_{j}(\mu ; h)\left(\partial q_{j} / \partial \mu_{s}\right)=w_{s}+\partial \mathcal{L}_{\mu}(h) / \partial \mu_{s},
\end{gathered}
$$

holds for all $s=\overline{1,2}$ and $\mu, \mu^{(0)} \in \otimes_{j=1}^{n} \mathbb{S}_{j}^{1}$ with parameters $h \in \mathbb{R}^{n}$ being fixed. Thereby we have found a natural generalization of relationships (3.34) subject to the extended integral submanifold imbedding mapping $\pi_{h}: M_{h}^{n} \rightarrow T^{*}\left(\mathbb{R}^{n}\right)$ in the form (3.28).

Assume now that functions $w_{j}: \mathbb{C} \times \mathbb{C}^{n} \rightarrow \mathbb{C}, j=\overline{1, n}$, satisfy in general PicardFuchs equations like (3.35), having the following [3] algebraic solutions:

$$
w_{j}^{n_{j}}+\sum_{k=0}^{n_{j}-1} c_{j, k}(\lambda ; h) w_{j}^{k}=0,
$$

where $c_{j, k}: \mathbb{C} \times \mathbb{C}^{n} \rightarrow \mathbb{C}, k=\overline{0, n_{j}-1}, j=\overline{1, n}$, are some polynomials in $\lambda \in \mathbb{C}$. Each algebraic curve of (3.41) is known to be in general topologically equivalent 
due to the Riemann theorem [20] to some Riemannian surface $\Gamma_{h}^{(j)}$, of genus $g_{j} \in \mathbb{Z}_{+}$, $j=\overline{1, n}$. Thereby, one can realize the local diffeomorphism $\varrho: M_{h}^{n} \rightarrow \otimes_{j=1}^{n} \Gamma_{h}^{(j)}$, mapping homology group basis cycles $\sigma_{j}^{(h)} \subset M_{h}^{n}, j=\overline{1, n}$, into homology subgroup $H_{1}\left(\otimes_{j=1}^{n} \Gamma_{h}^{(j)} ; \mathbb{Z}\right)$ basis cycles $\sigma_{j}\left(\Gamma_{h}\right) \subset \Gamma_{h}^{(j)}, j=\overline{1, n}$, satisfying the following relationships:

$$
\varrho\left(\sigma_{j}^{(h)}\right)=\sum_{k=1}^{n} n_{j k} \sigma_{k}\left(\Gamma_{h}\right),
$$

where $n_{j k} \in \mathbb{Z}, k=\overline{1, j}$ and $j=\overline{1, n}$, are some fixed integers. Based on (3.42) and (3.37) one can write down, for instance, expressions (3.27) as follows:

$$
\gamma_{i}=\frac{1}{2 \pi} \sum_{j=1}^{n} n_{i j} \oint_{\sigma_{j}\left(\Gamma_{h}\right)} w_{j}(\lambda ; h) d \lambda,
$$

where $i=\overline{1, n}$. Subject to the evolution on $M_{h}^{n} \subset T^{*}\left(\mathbb{R}^{n}\right)$ one can easily obtain from (3.39) that

$$
d t_{i}=\sum_{j=1}^{n}\left(\partial w_{j}\left(\mu_{j} ; h\right) / \partial h_{i}\right) d \mu_{j}
$$

at $d h_{i}=0$ for all $i=\overline{1, n}$, giving rise evidently to a global $\tau$-parametrization of the set of circles $\otimes_{j=1}^{n} \mathbf{S}_{j}^{1} \subset \otimes_{j=1}^{n} \Gamma_{h}^{(j)}$, that is one can define some inverse algebraic functions to Abelian type integrals (3.37) as

$$
\mu=\mu(\tau ; h),
$$

where as before, $\tau=\left(t_{1}, t_{2}, \ldots, t_{n}\right) \in \mathbb{R}^{n}$ is an a vector of evolution parameters. Recalling now expressions (3.28) for integral submanifold mapping $\pi_{h}: M_{h}^{n} \rightarrow T^{*}\left(\mathbb{R}^{n}\right)$, one can at last write down final expressed by "quadratures" mappings for evolutions on $M_{h}^{n} \subset T^{*}\left(\mathbb{R}^{n}\right)$ as follows:

$$
q=q(\mu(\tau ; h))=\tilde{q}(\tau ; h), \quad p=p(\mu(\tau ; h))=\tilde{p}(\tau ; h),
$$

where obviously, a vector $(\tilde{q}, \tilde{p}) \in T^{*}\left(\mathbb{R}^{n}\right)$ is quasiperiodic in each variable $t_{i} \in \tau$, $i=\overline{1, n}$.

Theorem 10. Every completely integrable Hamiltonian system admitting an algebraic submanifold $M_{h}^{n} \subset T^{*}\left(\mathbb{R}^{n}\right)$ pessesses a separable canonical transformation (3.38) which is described by differential algebraic Picard-Fuchs type equations whose solution is a set of algebraic curves (3.41).

Therefore, the main ingredient of this scheme of integrability by quadratures is finding the Picard-Fuchs type equations (3.35) corresponding to the integral submanifold imbedding mapping (3.28) depending in general on $\mathbb{R}^{n} \ni h$-parameters for the case when the integral submanifold $M_{h}^{n} \subset T^{*}\left(\mathbb{R}^{n}\right)$ cannot be imbedded into the base space $\mathbb{R}^{n} \subset T^{*}\left(\mathbb{R}^{n}\right)$ of the phase space $T^{*}\left(\mathbb{R}^{n}\right)$. Based now on Theorem 8 one 
can find 1-forms $h_{j}^{(1)} \in \Lambda^{1}\left(T^{*}\left(\mathbb{R}^{n}\right)\right), j=\overline{1, n}$, enjoying the following identity on $T^{*}\left(\mathbb{R}^{n}\right)$ :

$$
\omega^{(2)}(q, p):=\sum_{j=1}^{n} d p_{j} \wedge d q_{j}=\sum_{j=1}^{n} d H_{j} \wedge h_{j}^{(1)} .
$$

The 1-forms $h_{j}^{(1)} \in \Lambda^{1}\left(T^{*}\left(\mathbb{R}^{n}\right)\right), j=\overline{1, n}$, possess the stated important property: pullbacked to the integral submanifold (3.10) gives rise to the global linearization

$$
\pi_{h}^{*} h_{j}^{(1)}:=\bar{h}_{j}^{(1)}=d t_{j}
$$

where $\bar{h}_{j}^{(1)} \in \Lambda^{1}\left(M_{h}^{n}\right)$, and $\pi_{h *} d / d t_{j}=K_{j} \cdot \pi_{h}$ for all $j=\overline{1, n}$. Expressions (2.2) combined with those in (3.44) give rise easily to the following set of relationships

$$
\bar{h}_{j}^{(1)}=\sum_{j=1}^{n}\left(\partial w_{j}\left(\mu_{j} ; h\right) / \partial h_{i}\right) d \mu_{j}
$$

at $d h_{j}=0$ for all $j=\overline{1, n}$ on $M_{h}^{n} \simeq \otimes_{j=1}^{n} \mathbf{S}_{j}^{1} \subset \otimes_{j=1}^{n} \Gamma_{h}^{(j)}$ for all $j=\overline{1, n}$. Since we are interested in the integral submanifold imbedding mapping (3.25) being locally diffeomorphic in a neighborhood $U\left(M_{h}^{n}\right) \subset T^{*}\left(\mathbb{R}^{n}\right)$, the Jacobian $\operatorname{det}\|\partial q(\mu ; h) / \partial \mu\| \neq$ 0 almost everywhere in $U\left(M_{h}^{n}\right)$. On the other hand, as it was proved in [4], the set of 1-forms $\bar{h}_{j}^{(1)} \in \Lambda^{1}\left(M_{h}^{n}\right), j=\frac{h}{1, n}$, can be in general, represented in $U\left(M_{h}^{n}\right)$ as

$$
\bar{h}_{j}^{(1)}=\left.\sum_{k=1}^{n} \bar{h}_{j k}^{(1)}(q, p) d q_{k}\right|_{M_{h}^{n}},
$$

where $\bar{h}_{j k}^{(1)}: T^{*}\left(\mathbb{R}^{n}\right) \rightarrow \mathbb{R}, k, j=\overline{1, n}$, are some algebraic expressions of their arguments. Thereby, one easily finds from (3.50) and (3.49) that

$$
\partial w_{i}\left(\mu_{i} ; h\right) / \partial h_{j}=\sum_{k=1}^{n} \bar{h}_{j k}^{(1)}(q(\mu ; h), p(\mu ; h))\left(\partial q_{k}(\mu ; h) / \partial \mu_{i}\right)
$$

for all $i, j=\overline{1, n}$. Subject to $p$-variables in (3.51) we must, owing to (3.40), use the expressions

$$
\begin{aligned}
\sum_{j=1}^{n} p_{j}(\mu ; h)\left(\partial q_{j} / \partial \mu_{s}\right) & =w_{s}+\partial \mathcal{L}_{\mu}(h) / \partial \mu_{s}, \\
\partial \mathcal{L}_{\mu}(h) / \partial h_{j} & =\left.\left\langle p, \partial q / \partial h_{j}\right\rangle\right|_{M_{h}^{n}},
\end{aligned}
$$

being true for $s=\overline{1, n}$ and all $\mu \in \otimes_{j=1}^{n} \mathbb{S}_{j}, h \in \mathbb{R}^{n}$ in the neighborhood $U\left(M_{h}^{n}\right) \subset$ $T^{*}\left(\mathbb{R}^{n}\right)$ chosen before. Thereby, we arrived at the following form of equations (3.51):

$$
\partial w_{i}\left(\mu_{i} ; h\right) / \partial h_{j}=\bar{P}_{j i}(\mu, w ; h),
$$


where for all $i, j=\overline{1, n}$ expressions

$$
\bar{P}_{j i}(\mu, w ; h):=\sum_{k=1}^{n} \bar{h}_{j k}^{(1)}(q(\mu ; h), p(\mu ; h))\left(\partial q_{k} / \partial \mu_{i}\right)
$$

depend correspondingly only on $\Gamma_{h}^{(i)} \ni\left(\mu_{i}, w_{i}\right)$-variables for each $i \in\{\overline{1, n}\}$ and all $j=\overline{1, n}$. This condition can be evidently written as follows:

$$
\partial \bar{P}_{j i}(\mu, w ; h) / \partial \mu_{k}=0, \quad \partial \bar{P}_{j i}(\mu, w ; h) / \partial w_{k}=0
$$

for $j, i \neq k \in\{\overline{1, n}\}$ at almost all $\mu \in \otimes_{j=1}^{n} \mathbb{S}_{j}^{1}$ and $h \in \mathbb{R}^{n}$. The set of conditions (3.51) gives rise in general to a system of algebraic-differential equations subject to the imbedding mapping $\operatorname{pr} \pi_{h}: M_{h}^{n} \rightarrow \mathbb{R}^{n}$ defined analytically by (3.28) and the generating function (3.37). As a result of solving these equations we obtain evidently, owing to (3.53) and (3.55), the following system of Picard-Fuchs type equations:

$$
\partial w_{i}\left(\mu_{i} ; h\right) / \partial h_{j}=P_{j i}\left(\mu_{i}, w_{i} ; h\right)
$$

where, in general, mappings

$$
P_{j i}: \Gamma_{h}^{(i)} \times \mathbb{R}^{n} \rightarrow \mathbb{C}
$$

are some algebraic expressions. Since the set of algebraic curves (3.41) must enjoy the system (3.56), we can retrieve this set solving the Picard-Fuchs type equations (3.56). The latter gives rise due to (3.39) and (3.28) to the integrability of all flows on $M_{h}^{n} \subset T^{*}\left(\mathbb{R}^{n}\right)$ by quadratures as was mentioned in Section 1 .

Theorem 11. Let there be given a completely integrable Hamiltonian system on the coadjoint manifold $T^{*}\left(\mathbb{R}^{n}\right)$ whose integral submanifold $M_{h}^{n} \subset T^{*}\left(\mathbb{R}^{n}\right)$ is described by Picard-Fuchs type algebraic equations (3.56). The corresponding imbedding mapping $\pi_{h}: M_{h}^{n} \rightarrow T^{*}\left(\mathbb{R}^{n}\right)$ (3.28) is a solution of a compatibility condition subject to the differential-algebraic relationships (3.55) on the canonical transformations generating function (3.37).

To show that the scheme described above really leads to an algorithmic procedure of constructing the Picard-Fuchs type equations (3.56) and the corresponding integral submanifold imbedding mapping $\pi_{h}: M_{h}^{n} \rightarrow T^{*}\left(\mathbb{R}^{n}\right)$ in the form (3.28), we apply it below in Section 6 to some Hamiltonian systems including a so-called truncated Focker-Plank Hamiltonian system on the canonically symplectic cotangent space $T^{*}\left(\mathbb{R}^{n}\right)$. Making use of representations (3.21) and (3.28) and equation (3.31), we have shown above that the set of 1-forms (3.30) is reduced to the following purely differential-algebraic relations on $M_{h, \tau}^{2 n}$ :

$$
\partial w_{i}\left(\mu_{i} ; h\right) / \partial h_{j}=\mathbf{P}_{j i}\left(\mu_{i}, w_{i} ; h\right),
$$


which generalize similar relations from $[18,31]$, where the characteristic functions $\mathbf{P}_{j i}: T^{*}\left(M_{h}^{n}\right) \rightarrow \mathbb{R}, i, j=\overline{1, n}$, are defined as follows:

$$
\mathbf{P}_{j i}\left(\mu_{i}, w_{i} ; h\right):=\left.\overline{\mathbf{P}}_{j i}(\mu, w ; h)\right|_{M_{h}^{n}} .
$$

It is clear that the above set of purely differential-algebraic relationships (3.33) and (3.34) makes it possible to write explicitly some first order compatible differentialalgebraic equations, whose solution yields the first half of the desired imbedding (3.5) for the integral submanifold $M_{h}^{n} \subset M^{2 n}$ in an open neighborhood $M_{h, \tau}^{2 n} \subset M^{2 n}$. As a result of the above computations one can formulate the following main theorem.

Theorem 12. The imbedding (3.5) for the integral submanifold $M_{h}^{n} \subset M^{2 n}$ (compact and connected), parametrized by a regular parameter $h \in \mathcal{G}^{*}$, is an algebraic solution (up to diffeomorphism) to the set of characteristic Picard-Fuchs type equations (3.35) on $T^{*}\left(M_{h}^{n}\right)$, and can be represented in the general case [19] in the following algebraic-geometric form:

$$
w_{j}^{n_{j}}+\sum_{s=1}^{n} c_{j s}(\lambda ; h) w_{j}^{n_{j}-s}=0,
$$

where $c_{j s}: \mathbb{R} \times \mathcal{G}^{*} \rightarrow \mathbb{R}, s, j=\overline{1, n}$ are algebraic expressions, depending only on the functional structure of the original abelian Lie algebra $\mathcal{G}$ of invariants on $M^{2 n}$. In particular, if the right-hand side of the characteristic equations (3.35) is independent of $h \in G^{*}$, then this dependence will be linear in $h \in \mathcal{G}^{*}$.

It should be noted here that some ten years ago an attempt was made in $[18,19]$ to describe the explicit algebraic form of the Picard-Fuchs type equations (3.35) by means of straightforward calculations for the well-known completely integrable Kowalewskaya top Hamiltonian system. The idea suggested in $[18,19]$ was in some aspects very close to that devised independently and thoroughly analyzed in [11] which did not consider the explicit form of the algebraic curves (3.37) starting from an abelian Lie algebra $\mathcal{G}$ of invariants on a canonically symplectic phase space $M^{2 n}$.

As is well known, a set of algebraic curves (3.37), prescribed via the above algorithm, to a given a priori abelian Lie algebra $\mathcal{G}$ of invariants on the canonically symplectic phase space $M^{2 n}=T^{*}\left(\mathbb{R}^{n}\right)$ can be realized by means of a set of $n_{j^{-}}$ sheeted Riemannian surfaces $\Gamma_{h}^{n_{j}}, j=\overline{1, n}$, covering the corresponding real-valued cycles $\mathbb{S}_{j}^{1}, j=\overline{1, n}$, which generate the corresponding homology group $H_{1}\left(\mathbb{T}^{n} ; \mathbb{Z}\right)$ of the Arnold torus $\mathbb{T}^{n} \simeq \otimes_{j=1}^{n} \mathbb{S}_{j}^{1}$ diffeomorphic to the integral submanifold $M_{h}^{n} \subset M^{2 n}$.

Thus, upon solving the set of algebraic equations (3.37) with respect to the functions $w_{j}: \mathbb{S}_{j}^{1} \times \mathcal{G}^{*} \rightarrow \mathbb{R}, j=\overline{1, n}$, from (3.29) one obtains a vector parameter $\tau=\left(t_{1}, \ldots, t_{n}\right) \in \mathbb{R}^{n}$ on $M_{h}^{n}$ explicitly described by means of the following abelian 
type equations:

$$
t_{j}=\sum_{s=1}^{n} \int_{\mu_{s}^{0}}^{\mu_{s}} d \lambda \partial w_{s}(\lambda ; h) / \partial h_{j}=\sum_{s=1}^{n} \int_{\mu_{s}^{0}}^{\mu_{s}} d \lambda \overline{\mathbf{P}}_{j s}\left(\lambda, w_{s} ; h\right),
$$

where $j=\overline{1, n},\left(\mu^{0} ; h\right) \in\left(\otimes_{j=1}^{n} \Gamma_{h}^{n_{j}}\right) \times \mathcal{G}^{*}$. Using expression (3.28) and recalling that the generating function $S: M_{h}^{n} \times \mathbb{R}^{n} \rightarrow \mathbb{R}$ is a one-valued mapping on an appropriate covering space $\left(\bar{M}_{h}^{n} ; H_{1}\left(M_{h}^{n} ; \mathbb{Z}\right)\right)$, one can construct via the method of Arnold [4] the so called action-angle coordinates on $M_{h}^{n}$. Denote the basic oriented cycles on $M_{h}^{n}$ by $\sigma_{j} \subset M_{h}^{n}, j=\overline{1, n}$. These cycles together with their duals generate homology group $H_{1}\left(M_{h}^{n} ; \mathbb{Z}\right) \simeq H_{1}\left(\mathbb{T}^{n} ; \mathbb{Z}\right)=\oplus_{j=1}^{n} \mathbb{Z}_{j}$. By virtue of the diffeomorphism $M_{h}^{n} \simeq \otimes_{j=1}^{n} \mathbb{S}_{j}^{1}$ described above, there is a one-to-one correspondence between the basic cycles of $H_{1}\left(M_{h}^{n} ; \mathbb{Z}\right)$ and those on the algebraic curves $\Gamma_{h}^{n_{j}}, j=\overline{1, n}$, given by (3.37):

$$
\varrho: H_{1}\left(M_{h}^{n} ; \mathbb{Z}\right) \rightarrow \oplus_{j=1}^{n} \mathbb{Z}_{j} \sigma_{h, j},
$$

where $\sigma_{h, j} \subset \Gamma_{h}^{n_{j}}, j=\overline{1, n}$ are the corresponding real-valued cycles on the Riemann surfaces $\Gamma_{h}^{n_{j}}, j=\overline{1, n}$.

Assume that the following meanings of the mapping (3.37) are prescribed:

$$
\varrho\left(\sigma_{i}\right):=\oplus_{j=1}^{n} n_{i j} \sigma_{h, j}
$$

for each $i=\overline{1, n}$, where $n_{i j} \in \mathbb{Z}, i, j=\overline{1, n}$ - some fixed integers. Then following the Arnold construction $[4,18]$, one obtains the set of so-called action-variables on $M_{h}^{n} \subset M^{2 n}$ :

$$
\gamma_{j:}:=\frac{1}{2 \pi} \oint_{\sigma_{j}} d S=\sum_{s=1}^{n} n_{j s} \oint_{\sigma_{h, s}} d \lambda w_{s}(\lambda ; h),
$$

where $j=\overline{1, n}$. It is easy to show $[4,16]$ that expressions (3.41) naturally define an a. e. differentiable invertible mapping

$$
\xi: \mathcal{G}^{*} \rightarrow \mathbb{R}^{n}
$$

which enables one to treat the integral submanifold $M_{h}^{n}$ as a submanifold $M_{\gamma}^{n} \subset M^{2 n}$, where

$$
M_{\gamma}^{n}:=\left\{u \in M^{2 n}: \xi(h)=\gamma \in \mathbb{R}^{n}\right\} .
$$

But, as was demonstrated in $[18,32]$, functions $(3.43)$ do not in general generate a global foliation of the phase space $M^{2 n}$, as they are connected with both topological and analytical constraints. Since functions (3.41) are evidently also commuting invariants on $M^{2 n}$, one can define a further canonical transformation of the phase space $M^{2 n}$, generated by the following relationship on $M_{h, \tau}^{2 n}$ :

$$
\sum_{j=1}^{n} w_{j} d \mu_{j}+\sum_{j=1}^{n} \varphi_{j} d \gamma_{j}=d S(\mu ; \gamma)
$$


where $\varphi=\left(\varphi_{1}, \ldots, \varphi_{n}\right) \in \mathbb{T}^{n}$ are the so-called angle-variables on the torus $\mathbb{T}^{n} \simeq M_{h}^{n}$ and $S: M_{\gamma}^{n} \times \mathbb{R}^{n} \rightarrow \mathbb{R}$ is the corresponding generating function. Whence it follows easily from (3.28) and (3.38) that

$$
\begin{gathered}
\varphi_{j}:=\partial S(\mu ; \gamma) / \partial \gamma_{j}=\sum_{s=1}^{n} \partial S(\mu ; \gamma(h)) / \partial h_{s} \partial h_{s} / \partial \gamma_{j}=\sum_{s=1}^{n} t_{s} \omega_{s j}(\gamma), \\
\frac{1}{2 \pi} \oint_{\sigma_{j}} d \varphi_{k}=\delta_{j k},
\end{gathered}
$$

where $\Omega:=\left\{\omega_{s j}: \mathbb{R}^{n} \rightarrow \mathbb{R}, s, j=\overline{1, n}\right\}$ is the so-called [4] frequency matrix, which is a. e. invertible on the integral submanifold $M_{\gamma}^{n} \subset M^{2 n}$. As an evident result of (3.45), we claim that the evolution of any vector field $K_{a} \in \Gamma\left(M^{2 n}\right)$ for $a \in \mathcal{G}$ on the integral submanifold $M_{\gamma}^{n} \subset M^{2 n}$ is quasiperiodic with a set of frequencies generated by the matrix $\Omega \stackrel{\text { a. e. }}{\in}$ Aut $\left(\mathbb{R}^{n}\right)$ defined above. As examples showing the effectiveness of the above method of construction of integral submanifold imbeddings for abelian integrable Hamiltonian systems, one can verify the Liouville-Arnold integrability of all Henon-Heiles and Neumann type systems described in detail in [21,22]; however, we shall not dwell on this here.

\section{INTEGRAL SUBMANIFOLD IMBEDDING PROBLEM FOR A NONABELIAN LIE ALGEBRA OF INVARIANTS}

We shall assume below that there is given a Hamiltonian vector field $K \in \Gamma\left(M^{2 n}\right)$ on the canonically symplectic phase space $M^{2 n}=T^{*}\left(\mathbb{R}^{n}\right), n \in \mathbb{Z}_{+}$, which is endowed with a nonabelian Lie algebra $\mathcal{G}$ of invariants, satisfying all the conditions of the Mishchenko-Fomenko Theorem 6, that is

$$
\operatorname{dim} \mathcal{G}+\operatorname{rank} \mathcal{G}=\operatorname{dim} M^{2 n} .
$$

Then, as was proved above, an integral submanifold $M_{h}^{r} \subset M^{2 n}$ at a regular element $h \in \mathcal{G}^{*}$ is rank $\mathcal{G}=r$-dimensional and diffeomorphic (when compact and connected) to the standard $r$-dimensional torus $\mathbb{T}^{r} \simeq \otimes_{j=1}^{r} \mathbb{S}_{j}^{1}$. It is natural to ask the following question: How does one construct the corresponding integral submanifold imbedding

$$
\pi_{h}: M_{h}^{r} \rightarrow M^{2 n},
$$

which characterizes all possible orbits of the dynamical system $K \in \Gamma\left(M^{2 n}\right)$ ?

Having gained some experience in constructing the imbedding (4.2) in the case of the abelian Liouville-Arnold theorem on integrability by quadratures, we proceed below to study the integral submanifold $M_{h}^{r} \subset M^{2 n}$ by means of Cartan's theory $[3,12,16,22]$ of the integrable ideals in the Grassmann algebra $\Lambda\left(M^{2 n}\right)$. Let $\mathcal{I}\left(\mathcal{G}^{*}\right)$ be an ideal in $\Lambda\left(M^{2 n}\right)$, generated by independent differentials $d H_{j} \in \Lambda^{1}\left(M^{2 n}\right), j=\overline{1, k}$, in an open neighborhood $U\left(M_{h}^{r}\right)$, where by definition, $r=\operatorname{dim} \mathcal{G}$. The ideal $\mathcal{I}\left(\mathcal{G}^{*}\right)$ is obviously Cartan integrable [16,23] with the integral submanifold $M_{h}^{r} \subset M^{2 n}$ (at a 
regular element $\left.h \in \mathcal{G}^{*}\right)$, on which it vanishes, that is $\pi_{h}^{*} \mathcal{I}\left(\mathcal{G}^{*}\right)=0$. The dimension $\operatorname{dim} M_{h}^{r}=\operatorname{dim} M^{2 n}-\operatorname{dim} \mathcal{G}=r=\operatorname{rank} \mathcal{G}$ due to condition (4.1) imposed on the Lie algebra $\mathcal{G}$. It is useful to note here that, owing to the inequality $r \leq k$ for the $\operatorname{rank} \mathcal{G}$, one readily obtains from (3.1) that $\operatorname{dim} \mathcal{G}=k \geq n$. Since each base element $H_{j} \in \mathcal{G}, j=\overline{1, k}$, generates a symplectically dual vector field $K_{j} \in \Gamma\left(M^{2 n}\right), j=\overline{1, k}$, one can try to study the corresponding differential system $K(\mathcal{G})$ which is also Cartan integrable in the entire open neighborhood $U\left(M_{h}^{r}\right) \subset M^{2 n}$. Denote the corresponding dimension of the integral submanifold by $\operatorname{dim} M_{h}^{k}=\operatorname{dim} K(\mathcal{G})=k$. Consider now an abelian differential system $K\left(\mathcal{G}_{h}\right) \subset K(\mathcal{G})$, generated by the Cartan subalgebra $\mathcal{G}_{h} \subset$ $\mathcal{G}$ and its integral submanifold $\bar{M}_{h}^{r} \subset U\left(M_{h}^{r}\right)$. Since the Lie subgroup $G_{h}=\exp \mathcal{G}_{h}$ acts on the integral submanifold $M_{h}^{r}$ invariantly (see Section 2) and $\operatorname{dim} \bar{M}_{h}^{r}=\operatorname{rank}$ $\mathcal{G}=r$, it follows that $\bar{M}_{h}^{r}=M_{h}^{r}$. On the other hand, the system $K\left(\mathcal{G}_{h}\right) \subset K(\mathcal{G})$ by definition means that the integral submanifold $M_{h}^{r}$ is an invariant part of the integral submanifold $M_{h}^{k} \subset U\left(M_{h}^{r}\right)$ with respect to the Lie group $G=\exp \mathcal{G}$-action on $M_{h}^{k}$. In this case one has the following result.

Lemma 5. There exist just $(n-r) \in \mathbb{Z}_{+}$vector fields $\tilde{F}_{j} \in K(\mathcal{G}) / K\left(\mathcal{G}_{h}\right), j=$ $\overline{1, n-r}$, for which

$$
\omega^{(2)}\left(\tilde{F}_{i}, \tilde{F}_{j}\right)=0
$$

on $U\left(M_{h}^{r}\right)$ for all $i, j=\overline{1, n-r}$.

Proof. It is obvious that the matrix $\omega(\tilde{K}):=\left\{\omega^{(2)}\left(\tilde{K}_{i}, \tilde{K}_{j}\right): i, j=\overline{1, k}\right\}$ has on $U\left(M_{h}^{r}\right)$ the rank $\omega(\tilde{K})=k-r$, since $\operatorname{dim}_{\mathbb{R}} \operatorname{ker}\left(\pi_{h}^{*} \omega^{(2)}\right)=\operatorname{dim}_{\mathbb{R}}\left(\pi_{h *} K\left(\mathcal{G}_{h}\right)\right)=r$ on $M_{h}^{r}$ at the regular element $h \in \mathcal{G}^{*}$. Let us now complexify the tangent space $T\left(U\left(M_{h}^{r}\right)\right)$ using its even dimensionality. Whence one can easily deduce that on $U\left(M_{h}^{r}\right)$ there exist just $(n-r) \in \mathbb{Z}_{+}$vectors (not vector fields!) $\tilde{K}_{j}^{\mathbb{C}} \in K^{\mathbb{C}}(\mathcal{G}) / K^{\mathbb{C}}\left(\mathcal{G}_{h}\right), j=\overline{1, n-r}$, from the complexified [24] factor space $K^{\mathbb{C}}(\mathcal{G}) / K^{\mathbb{C}}\left(\mathcal{G}_{h}\right)$. To show this, let us reduce the skew-symmetric matrix $\omega(\tilde{K}) \in \operatorname{Hom}\left(\mathbb{R}^{k-r}\right)$ to its self-adjoint equivalent $\omega\left(\tilde{K}^{\mathbb{C}}\right) \in \operatorname{Hom}\left(\mathbb{C}^{n-r}\right)$, having taken into account that $\operatorname{dim}_{\mathbb{R}} \mathbb{R}^{k-r}=\operatorname{dim}_{\mathbb{R}} \mathbb{R}^{k+r-2 r}=$ $\operatorname{dim}_{\mathbb{R}} \mathbb{R}^{2(n-r)}=\operatorname{dim}_{\mathbb{C}} \mathbb{C}^{n-r}$. Let now $f_{j}^{\mathbb{C}} \in \mathbb{C}^{n-r}, j=\overline{1, n-r}$, be eigenvectors of the nondegenerate self-adjoint matrix $\omega\left(\tilde{K}^{\mathbb{C}}\right) \in \operatorname{Hom}\left(\mathbb{C}^{n-r}\right)$, that is

$$
\omega\left(\tilde{K}^{\mathbb{C}}\right) f_{j}^{\mathbb{C}}=\tilde{\lambda}_{j} f_{j}^{\mathbb{C}},
$$

where $\tilde{\lambda}_{j} \in \mathbb{R}, j=\overline{1, n-r}$, and for all $i, j=\overline{1, n-r},\left\langle f_{i}^{\mathbb{C}}, f_{j}^{\mathbb{C}}\right\rangle=\delta_{i, j}$. The above obviously means that in the basis $\left\{f_{j}^{\mathbb{C}} \in K^{\mathbb{C}}(\mathcal{G}) / K^{\mathbb{C}}\left(\mathcal{G}_{h}\right): j=\overline{1, n-r}\right\}$ the matrix $\omega\left(\tilde{K}^{\mathbb{C}}\right) \in \operatorname{Hom}\left(\mathbb{C}^{n-r}\right)$ is strictly diagonal and representable as

$$
\omega\left(\tilde{K}^{\mathbb{C}}\right)=\sum_{j=1}^{n-r} \tilde{\lambda}_{j} f_{j}^{\mathbb{C}} \otimes_{\mathbb{C}} f_{j}^{\mathbb{C}},
$$


where $\otimes_{\mathbb{C}}$ is the usual Kronecker tensor product of vectors from $\mathbb{C}^{n-r}$. Owing to the construction of the complexified matrix $\omega\left(\tilde{K}^{\mathbb{C}}\right) \in \operatorname{Hom}\left(\mathbb{C}^{n-r}\right)$, one sees that the space $K^{\mathbb{C}}(\mathcal{G}) / K^{\mathbb{C}}\left(G_{h}\right) \simeq \mathbb{C}^{n-r}$ carries a Kähler structure [24] with respect to which the following expressions

$$
\omega(\tilde{K})=\operatorname{Im} \omega(\tilde{K}), \quad\langle\cdot, \cdot\rangle_{\mathbb{R}}=\operatorname{Re}\langle\cdot, \cdot\rangle
$$

hold. Making use now of the representation (4.5) and expressions (4.6), one can find vector fields $\tilde{F}_{j} \in K(\mathcal{G}) / K\left(\mathcal{G}_{h}\right), j=\overline{1, n-r}$, such that

$$
\omega(\tilde{F})=\operatorname{Im} \omega\left(\tilde{F}^{\mathbb{C}}\right)=J
$$

holds on $U\left(M_{h}^{r}\right)$, where $J \in \operatorname{Sp}\left(\mathbb{C}^{n-r}\right)$ is the standard symplectic matrix, satisfying the complex structure [24] identity $J^{2}=-I$. By virtue of the normalization conditions $\left\langle f_{j}^{\mathbb{C}}, f_{j}^{\mathbb{C}}\right\rangle=\delta_{i, j}$, for all $i, j=\overline{1, n-r}$, one easily infers from $(3.7)$ that $\omega^{(2)}\left(\tilde{F}_{i}, \tilde{F}_{j}\right)=0$ for all $i, j=\overline{1, n-r}$, where by definition

$$
\tilde{F}_{j}:=\operatorname{Re} \tilde{F}_{j}^{\mathbb{C}}
$$

for all $j=\overline{1, n-r}$, and this proves the lemma.

Assume now that the Lie algebra $\mathcal{G}$ of invariants on $M^{2 n}$ has been split into a direct sum of subspaces as

$$
\mathcal{G}=\mathcal{G}_{h} \oplus \tilde{\mathcal{G}}_{h},
$$

where $\mathcal{G}_{h}$ is the Cartan subalgebra at a regular element $h \in \mathcal{G}^{*}$ (being abelian) and $\tilde{\mathcal{G}}_{h} \simeq \mathcal{G} / \mathcal{G}_{h}$ is the corresponding complement to $\mathcal{G}_{h}$. Denote a basis of $\mathcal{G}_{h}$ as $\left\{\bar{H}_{i} \in\right.$ $\left.\mathcal{G}_{h}: i=\overline{1, r}\right\}$, where $\operatorname{dim} \mathcal{G}_{h}=\operatorname{rank} \mathcal{G}=k \in \mathbb{Z}_{+}$, and correspondingly, a basis of $\tilde{\mathcal{G}}_{h}$ as $\left\{\tilde{H}_{j} \in \tilde{\mathcal{G}}_{h} \simeq \mathcal{G} / \mathcal{G}_{h}: j=\overline{1, k-r}\right\}$. Then, owing to the results of Section 2, the following relationships hold:

$$
\left\{\bar{H}_{i}, \bar{H}_{j}\right\}=0, \quad h\left(\left\{\bar{H}_{i}, \tilde{H}_{s}\right\}\right)=0
$$

in the open neighborhood $U\left(M_{h}^{r}\right) \subset M^{2 n}$ for all $i, j=\overline{1, r}$ and $s=\overline{1, k-r}$. We have as yet had nothing to say of expressions $h\left(\left\{\tilde{H}_{s}, \tilde{H}_{m}\right\}\right)$ for $s, m=\overline{1, k-r}$. Making use of the representation (4.8) for our vector fields (if they exist) $\tilde{F}_{j} \in K(\mathcal{G}) / K\left(\mathcal{G}_{h}\right)$, $j=\overline{1, n-r}$, one can write the following expansion:

$$
\tilde{F}_{i}=\sum_{j=1}^{k-r} c_{j i}(h) \tilde{K}_{j},
$$

where $i_{\tilde{K}_{j}} \omega^{(2)}:=-d \tilde{H}_{j}, c_{j i}: \mathcal{G}^{*} \rightarrow \mathbb{R}, i=\overline{1, n-r}, j=\overline{1, k-r}$, are real-valued functions on $\mathcal{G}^{*}$, being defined uniquely as a result of (4.11). Whence it clearly follows that there exist invariants $\tilde{f}_{s}: U\left(M_{h}^{r}\right) \rightarrow \mathbb{R}, s=\overline{1, n-r}$, such that

$$
-i_{\tilde{F}_{s}} \omega^{(2)}=\sum_{j=1}^{k-r} c_{j s}(h) d \tilde{H}_{j}:=d \tilde{f}_{s},
$$


where $\tilde{f}_{s}=\sum_{j=1}^{k-r} c_{j s}(h) \tilde{H}_{j}, s=\overline{1, n-r}$, holds on $U\left(M_{h}^{r}\right)$.

To proceed further, let us look at the following identity which is similar to (3.2):

$$
\left(\otimes_{j=1}^{r} i_{\bar{K}_{j}}\right)\left(\otimes_{s=1}^{n-r} i_{\tilde{F}_{s}}\right)\left(\omega^{(2)}\right)^{n+1}=0= \pm(n+1) !\left(\wedge_{j=1}^{r} d \bar{H}_{j}\right)\left(\wedge_{s=1}^{n-r} d \tilde{f}_{s}\right) \wedge \omega^{(2)} \text {, }
$$

on $U\left(M_{h}^{r}\right)$. Whence, the following result is easily obtained using Cartan theory $[3,16]$ :

Lemma 6. The symplectic structure $\omega^{(2)} \in \Lambda^{2}\left(U\left(M_{h}^{r}\right)\right)$ has the following canonical representation:

$$
\left.\omega^{(2)}\right|_{U\left(M_{h}^{r}\right)}=\sum_{j=1}^{r} d \bar{H}_{j} \wedge \bar{h}_{j}^{(1)}+\sum_{s=1}^{n-r} d \tilde{f}_{s} \wedge \tilde{h}_{s}^{(1)},
$$

where $\bar{h}_{j}^{(1)}, \tilde{h}_{s}^{(1)} \in \Lambda^{1}\left(U\left(M_{h}^{r}\right)\right), j=\overline{1, r}, s=\overline{1, n-r}$.

Expression (4.14) obviously means, that on $U\left(M_{h}^{r}\right) \subset M^{2 n}$ the differential 1-forms $\bar{h}_{j}^{(1)}, \tilde{h}_{s}^{(1)} \in \Lambda^{1}\left(U\left(M_{h}^{r}\right)\right), j=\overline{1, r}, s=\overline{1, n-r}$, are independent together with exact 1 -forms $d \bar{H}_{j}, j=\overline{1, r}$, and $d \tilde{f}_{s}, s=\overline{1, n-r}$. Since $d \omega^{(2)}=0$ on $M^{2 n}$ identically, from (4.14) one obtains that the differentials $d \bar{h}_{j}^{(1)}, d \tilde{h}_{s}^{(1)} \in \Lambda^{2}\left(U\left(M_{h}^{r}\right)\right), j=\overline{1, r}$, $s=\overline{1, n-r}$, belong to the ideal $\mathcal{I}\left(\tilde{G}_{h}\right) \subset \mathcal{I}\left(\mathcal{G}^{*}\right)$, generated by exact forms $d \tilde{f}_{s}$, $s=\overline{1, n-r}$, and $d \bar{H}_{j}, j=\overline{1, r}$, for all regular $h \in \mathcal{G}^{*}$. Consequently, one obtains the following analog of the Galisau-Reeb Theorem 8.

Theorem 13. Let a Lie algebra $\mathcal{G}$ of invariants on the symplectic space $M^{2 n}$ be nonabelian and satisfy the Mishchenko-Fomenko condition (4.1). At a regular element $h \in \mathcal{G}^{*}$ in some open neighborhood $U\left(M_{h}^{r}\right)$ of the integral submanifold $M_{h}^{r} \subset M^{2 n}$ there exist differential 1 -forms $\bar{h}_{j}^{(1)}, j=\overline{1, n}$, and $\tilde{h}_{s}^{(1)}, s=\overline{1, n-r}$, satisfying the following properties:

(i) $\left.\omega^{(2)}\right|_{U\left(M_{h}^{r}\right)}=\sum_{j=1}^{r} d \bar{H}_{j} \wedge \bar{h}_{j}^{(1)}+\sum_{s=1}^{n-r} d \tilde{f}_{s} \wedge \tilde{h}_{s}^{(1)}$ where $\bar{H}_{j} \in \mathcal{G}, j=\overline{1, r}$, is a basis of the Cartan subalgebra $\mathcal{G}_{h} \subset \mathcal{G}$ (being abelian), and $\tilde{f}_{s} \in \mathcal{G}, s=\overline{1, n-r}$, are invariants from the complementary space $\tilde{\mathcal{G}}_{h} \simeq \mathcal{G} / \mathcal{G}_{h}$;

(ii) The 1-forms $\bar{h}_{j}^{(1)} \in \Lambda^{1}\left(U\left(M_{h}^{r}\right)\right), j=\overline{1, r}$, and $\tilde{h}_{s}^{(1)} \in \Lambda^{1}\left(U\left(M_{h}^{r}\right)\right), s=\overline{1, n-r}$, are exact on $M_{h}^{r}$ and satisfy the equations: $\bar{h}_{j}^{(1)}\left(\bar{K}_{i}\right)=\delta_{i, j}$ for all $i, j=\overline{1, r}$, $\bar{h}_{j}^{(1)}\left(\tilde{F}_{s}\right)=0$ and $\tilde{h}_{s}^{(1)}\left(\bar{K}_{j}\right)=0$ for all $j=\overline{1, r}, s=\overline{1, n-r}$, and $\tilde{h}_{s}^{(1)}\left(\tilde{F}_{m}\right)=\delta_{s, m}$ for all $s, m=\overline{1, n-r}$.

Proof. Obviously we need to prove only the last statement (ii). Making use of Theorem 13, one can find on the integral submanifold $M_{h}^{r} \subset M^{2 n}$ the differential 2-forms $d \bar{h}_{j}^{(1)} \in \Lambda^{2}\left(U\left(M_{h}^{r}\right)\right), j=\overline{1, r}$, and $d \tilde{h}_{s}^{(1)} \in \Lambda^{2}\left(U\left(M_{h}^{r}\right)\right), s=\overline{1, n-r}$, are identically vanishing. This means in particular, owing to the classical Poincaré lemma $[1,4,16]$, that there exist some exact 1-forms $d \bar{t}_{h, j} \in \Lambda^{1}\left(U\left(M_{h}^{r}\right)\right), j=\overline{1, r}$, and $d \tilde{t}_{h, s} \in$ $\Lambda^{1}\left(U\left(M_{h}^{r}\right)\right), s=\overline{1, n-r}$, where $\bar{t}_{h, j}: M_{h}^{r} \rightarrow \mathbb{R}, j=\overline{1, r}$, and $\tilde{t}_{h, s}: M_{h}^{r} \rightarrow \mathbb{R}$, 
$s=\overline{1, n-r}$, are smooth independent a. e. functions on $M_{h}^{r}$; they are one-valued on an appropriate covering of the manifold $M_{h}^{r} \subset M^{2 n}$ and supply global coordinates on the integral submanifold $M_{h}^{r}$. Using representation (4.14), one can easily obtain that

$$
-\left.i_{\bar{K}_{i}} \omega^{(2)}\right|_{U\left(M_{h}^{r}\right)}=\sum_{j=1}^{r} d \bar{H}_{j} \bar{h}_{j}^{(1)}\left(\bar{K}_{i}\right)+\sum_{s=1}^{n-r} d \tilde{f}_{s} \tilde{h}_{s}^{(1)}\left(\bar{K}_{i}\right)=d \bar{H}_{i}
$$

for all $i=\overline{1, r}$ and

$$
-\left.i_{\tilde{F}_{m}} \omega^{(2)}\right|_{U\left(M_{h}^{r}\right)}=\sum_{j=1}^{r} d \bar{H}_{j} \bar{h}_{j}^{(1)}\left(\tilde{F}_{m}\right)+\sum_{s=1}^{n-r} d \tilde{f}_{s} \tilde{h}_{s}^{(1)}\left(\tilde{F}_{m}\right)=d \tilde{f}_{m}
$$

for all $m=\overline{1, n-r}$. Whence, from (4.15) it follows on that on $U\left(M_{h}^{r}\right)$,

$$
\bar{h}_{j}^{(1)}\left(\bar{K}_{i}\right)=\delta_{i, j}, \quad \tilde{h}_{s}^{(1)}\left(\bar{K}_{i}\right)=0
$$

for all $i, j=\overline{1, r}$ and $s=\overline{1, n-r}$, and similarly, from (4.16) it follows that on $U\left(M_{h}^{r}\right)$,

$$
\bar{h}_{j}^{(1)}\left(\tilde{F}_{m}\right)=0, \quad \tilde{h}_{s}^{(1)}\left(\tilde{F}_{m}\right)=0
$$

for all $j=\overline{1, r}$ and $s, m=\overline{1, n-r}$. Thus the theorem is proved.

Having now defined global evolution parameters $t_{j}: M^{2 n} \rightarrow \mathbb{R}, j=\overline{1, r}$, of the corresponding vector fields $\bar{K}_{j}=d / d t_{j}, j=\overline{1, r}$, and local evolution parameters $\tilde{t}_{s}$ : $M^{2 n} \cap U\left(M_{h}^{r}\right) \rightarrow \mathbb{R}, s=\overline{1, n-r}$, of the corresponding vector fields $\left.\tilde{F}_{s}\right|_{U\left(M_{h}^{r}\right)}:=d / d \tilde{t}_{s}$, $s=\overline{1, n-r}$, one can easily see from (4.18) that the equalities

$$
\left.t_{j}\right|_{U\left(M_{h}^{r}\right)}=\bar{t}_{j},\left.\quad \tilde{t}_{s}\right|_{U\left(M_{h}^{r}\right)}=\tilde{t}_{h, s}
$$

hold for all $j=\overline{1, r}, s=\overline{1, n-r}$, up to constant normalizations. Thereby, one can develop a new method, similar to that of Section 3, for studying the integral submanifold imbedding problem in the case of the nonabelian Liouville-Arnold integrability theorem.

Before starting, it is interesting to note that the system of invariants

$$
\mathcal{G}_{\tau}:=\mathcal{G}_{h} \oplus \operatorname{span}_{\mathbb{R}}\left\{\tilde{f}_{s} \in \mathcal{G} / \mathcal{G}_{h}: s=\overline{1, n-r}\right\}
$$

constructed above, comprises a new involutive (abelian) complete algebra $\mathcal{G}_{\tau}$, to which one can apply the abelian Liouville-Arnold theorem on integrability by quadratures and the integral submanifold imbedding theory devised in Section 3, in order to obtain exact solutions by means of algebraic-analytical expressions. Namely, the following corollary holds.

Corollary 3. Assume that a nonabelian Lie algebra $\mathcal{G}$ satisfies the MishchenkoFomenko condition (4.1) and $M_{h}^{r} \subset M^{2 n}$ is its integral submanifold (compact and connected) at a regular element $h \in \mathcal{G}^{*}$, is diffeomorphic to the standard torus $\mathbb{T}^{r} \simeq M_{h, \tau}^{n}$. 
Assume also that the dual complete abelian algebra $\mathcal{G}_{\tau}\left(\operatorname{dim} \mathcal{G}_{\tau}=n=1 / 2 \operatorname{dim} M^{2 n}\right)$ of independent invariants constructed above is globally defined. Then its integral submanifold $M_{h, \tau}^{n} \subset M^{2 n}$ is diffeomorphic to the standard torus $\mathbb{T}^{n} \simeq M_{h, \tau}^{n}$, and contains the torus $\mathbb{T}^{r} \simeq M_{h}^{r}$ as a direct product with some completely degenerate torus $\mathbb{T}^{n-r}$, that is $M_{h, \tau}^{n} \simeq M_{h}^{r} \times \mathbb{T}^{n-r}$.

Thus, having successfully applied the algorithm of Section 3 to the algebraicanalytical characterization of integral submanifolds of a nonabelian Liouville-Arnold integrable Lie algebra $\mathcal{G}$ of invariants on the canonically symplectic manifold $M^{2 n} \simeq$ $T^{*}\left(\mathbb{R}^{n}\right)$, one can produce a wide class of exact solutions represented by quadratures - which is just what we set out to find. At this point it is necessary to note that up to now the (dual to $\mathcal{G}$ ) abelian complete algebra $\mathcal{G}_{\tau}$ of invariants at a regular $h \in \mathcal{G}^{*}$ was constructed only in some open neighborhood $U\left(M_{h}^{r}\right)$ of the integral submanifold $M_{h}^{r} \subset M^{2 n}$. As mentioned before, the global existence of the algebra $\mathcal{G}_{\tau}$ strongly depends on the possibility of extending these invariants to the entire manifold $M^{2 n}$. This possibility is in 1-1 correspondence with the existence of a global complex structure [24] on the reduced integral submanifold $\tilde{M}_{h, \tau}^{2(n-r)}:=M_{h}^{k} / G_{h}$, induced by the reduced symplectic structure $\pi_{\tau}^{*} \omega^{(2)} \in \Lambda^{2}\left(M_{h}^{k} / G_{h}\right)$, where $\pi_{\tau}: M_{h}^{k} \rightarrow M^{2 n}$ is the imbedding for the integrable differential system $K(\mathcal{G}) \subset \Gamma\left(M^{2 n}\right)$, introduced above. If this is the case, the resulting complexified manifold ${ }^{\mathbb{C}} \tilde{M}_{h, \tau}^{n-r} \simeq \tilde{M}_{h, \tau}^{2(n-r)}$ will be endowed with a Kählerian structure, which makes it possible to produce the dual abelian algebra $\mathcal{G}_{\tau}$ as a globally defined set of invariants on $M^{2 n}$. This problem will be analyzed in more detail in Section 6.

\section{EXAmples}

Below we consider some examples of nonabelian Liouville-Arnold integrability by quadratures covered by Theorem 6 .

Example 2 (Point vortices in the plane). Consider $n \in \mathbb{Z}_{+}$point vortices on the plane $\mathbb{R}^{2}$, described by the Hamiltonian function

$$
H=-\frac{1}{2 \pi} \sum_{i \neq j=1}^{n} \xi_{i} \xi_{j} \ln \left\|q_{i}-p_{j}\right\|
$$

with respect to the following partially canonical symplectic structure on $M^{2 n} \simeq$ $T^{*}\left(\mathbb{R}^{n}\right)$ :

$$
\omega^{(2)}=\sum_{j=1}^{n} \xi_{j} d p_{j} \wedge d q_{j}
$$


where $\left(p_{j}, q_{j}\right) \in \mathbb{R}^{2}, j=\overline{1, n}$, are coordinates of the vortices in $\mathbb{R}^{2}$. There exist three additional invariants

$$
\begin{gathered}
P_{1}=\sum_{j=1}^{n} \xi_{j} q_{j}, \quad P_{2}=\sum_{j=1}^{n} \xi_{j} p_{j}, \\
P=\frac{1}{2} \sum_{j=1}^{n} \xi_{j}\left(q_{j}^{2}+p_{j}^{2}\right),
\end{gathered}
$$

satisfying the following Poisson bracket conditions:

$$
\begin{gathered}
\left\{P_{1}, P_{2}\right\}=-\sum_{j=1}^{n} \xi_{j}, \quad\left\{P_{1}, P\right\}=-P_{2}, \quad\left\{P_{2}, P\right\}=P_{1}, \\
\{P, H\}=0=\left\{P_{j}, H\right\} .
\end{gathered}
$$

It is evident, that invariants (5.1) and (5.3) comprise on $\sum_{j=1}^{n} \xi_{j}=0$ a fourdimensional Lie algebra $\mathcal{G}$, whose $\operatorname{rank} \mathcal{G}=2$. Indeed, assume that a regular vector $h \in \mathcal{G}^{*}$ is chosen, and parametrized by real values $h_{j} \in \mathbb{R}, j=\overline{1,4}$, where

$$
h\left(P_{i}\right)=h_{i}, \quad h(P)=h_{3}, \quad h(H)=h_{4},
$$

and $i=\overline{1,2}$. Then, one can easily verify that the element

$$
Q_{h}=\left(\sum_{j=1}^{n} \xi_{j}\right) P-\sum_{i=1}^{n} h_{i} P_{i}
$$

belongs to the Cartan Lie subalgebra $\mathcal{G}_{h} \subset \mathcal{G}$, that is

$$
h\left(\left\{Q_{h}, P_{i}\right\}\right)=0, \quad h\left(\left\{Q_{h}, P\right\}\right)=0 .
$$

Since $\left\{Q_{h}, H\right\}=0$ for all values $h \in \mathcal{G}^{*}$, we claim that $\mathcal{G}_{h}=\operatorname{span}_{\mathbb{R}}\left\{H, Q_{h}\right\}$ - the Cartan subalgebra of $\mathcal{G}$. Thus, $\operatorname{rank} \mathcal{G}=\operatorname{dim} \mathcal{G}_{h}=2$, and it comes right away that the condition (4.1)

$$
\operatorname{dim} M^{2 n}=2 n=\operatorname{rank} \mathcal{G}+\operatorname{dim} \mathcal{G}=6
$$

holds only if $n=3$. Thereby, the following theorem is proved.

Theorem 14. The three-vortex problem (5.1) on the plane $\mathbb{R}^{2}$ is nonabelian Liouville-Arnold integrable by quadratures on the phase space $M^{6} \simeq T^{*}\left(\mathbb{R}^{3}\right)$ with the symplectic structure (5.2).

As a result, the corresponding integral submanifold $M_{h}^{2} \subset M^{6}$ is two-dimensional and diffeomorphic (when compact and connected) to the torus $\mathbb{T}^{2} \simeq M_{h}^{2}$, on which the motions are quasiperiodic functions of the evolution parameter.

Concerning Corollary 3, the dynamical system (5.1) is also abelian LiouvilleArnold integrable with an extended integral submanifold $M_{h, \tau}^{3} \subset M^{6}$, which can be found via the scheme suggested in Section 4. Using simple calculations, one obtains an additional invariant $Q=\left(\sum_{j=1}^{3} \xi_{j}\right) P-\sum_{i=1}^{3} P_{i}^{2} \notin \mathcal{G}$, which commutes with 
$H$ and $P$ of $\mathcal{G}_{h}$. Therefore, there exists a new complete dual abelian algebra $\mathcal{G}_{\tau}=$ $\operatorname{span}_{\mathbb{R}}\{Q, P, H\}$ of independent invariants on $M^{6}$ with $\operatorname{dim} \mathcal{G}_{\tau}=3=1 / 2 \operatorname{dim} M^{6}$, whose integral submanifold $M_{h, \tau}^{3} \subset M^{6}$ (when compact and connected) is diffeomorphic to the torus $\mathbb{T}^{3} \simeq M_{h}^{2} \times \mathbb{S}^{1}$.

Note also here that the above additional invariant $Q \in \mathcal{G}_{\tau}$ can be naturally extended to the case of an arbitrary number $n \in \mathbb{Z}_{+}$of vortices as follows: $Q=\left(\sum_{j=1}^{n} \xi_{j}\right) P-$ $\sum_{i=1}^{n} P_{i}^{2} \in \mathcal{G}_{\tau}$, which obviously also commutes with invariants (5.1) and (5.3) on the entire phase space $M^{2 n}$.

Example 3 (A material point motion in a central field). Consider the motion of a material point in the space $\mathbb{R}^{3}$ under a central potential field whose Hamiltonian

$$
H=\frac{1}{2} \sum_{j=1}^{3} p_{j}^{2}+Q(\|q\|),
$$

contains a central field $Q: \mathbb{R}_{+} \rightarrow \mathbb{R}$. The motion takes place in the canonical phase space $M^{6}=T^{*}\left(\mathbb{R}^{3}\right)$, and possesses three additional invariants:

$$
P_{1}=p_{2} q_{3}-p q, \quad P_{2}=p_{3} q_{1}-p_{1} q_{3}, \quad P_{3}=p_{1} q_{2}-p_{2} q_{1},
$$

satisfying the following Poisson bracket relations:

$$
\left\{P_{1}, P_{2}\right\}=P_{3}, \quad\left\{P_{3}, P_{1}\right\}=P_{2}, \quad\left\{P_{2}, P_{3}\right\}=P_{1} .
$$

Since $\left\{H, P_{j}\right\}=0$ for all $j=\overline{1,3}$, one sees that the problem under consideration has a four-dimensional Lie algebra $\mathcal{G}$ of invariants, isomorphic to the classical rotation Lie algebra so $(3) \times \mathbb{R} \simeq \mathcal{G}$. Let us show that at a regular element $h \in \mathcal{G}^{*}$ the Cartan subalgebra $\mathcal{G}_{h} \subset \mathcal{G}$ has the dimension $\operatorname{dim} \mathcal{G}_{h}=2=\operatorname{rank} \mathcal{G}$. Indeed, one easily verifies that the invariant

$$
P_{h}=\sum_{j=1}^{3} h_{j} P_{j}
$$

belongs to the Cartan subalgebra $\mathcal{G}$, that is

$$
\left\{H, P_{h}\right\}=0, \quad h\left(\left\{P_{h}, P_{j}\right\}\right)=0
$$

for all $j=\overline{1,3}$. Thus, as the Cartan subalgebra $\mathcal{G}_{h}=\operatorname{span}_{\mathbb{R}}\left\{H\right.$ and $\left.P_{h} \subset \mathcal{G}\right\}$, one gets $\operatorname{dim} \mathcal{G}_{h}=2=\operatorname{rank} \mathcal{G}_{h}$, and the Mishchenko-Fomenko condition (4.1)

$$
\operatorname{dim} M^{6}=6=\operatorname{rank} \mathcal{G}+\operatorname{dim} \mathcal{G}=4+2
$$

holds. Hence one can prove its integrability by quadratures via the nonabelian Liouville Liouville-Arnold Theorem 6 and obtain the following theorem:

Theorem 15. It follows from Theorem 6 that the free material point motion in $\mathbb{R}^{3}$ is a completely integrable by quadratures dynamical system on the canonical symplectic phase space $M^{6}=T^{*}\left(\mathbb{R}^{3}\right)$. The corresponding integral submanifold $M_{h}^{2} \subset$ 
$M^{6}$ at a regular element $h \in \mathcal{G}^{*}$ (if compact and connected) is two-dimensional and diffeomorphic to the standard torus $\mathbb{T}^{2} \simeq M_{h}^{2}$.

Making use of the integration algorithm devised in Section 2 and 3, one can readily obtain the corresponding integral submanifold imbedding mapping $\pi_{h}: M_{h}^{2} \rightarrow M^{6}$ by means of algebraic-analytical expressions and transformations.

There are clearly many other interesting nonabelian Liouville-Arnold integrable Hamiltonian systems on canonically symplectic phase spaces that arise in applications, which can similarly be integrated using algebraic-analytical means. We hope to study several of these systems in detail elsewhere.

\section{EXISTENCE PROBLEM FOR A GLOBAL SET OF INVARIANTS}

It was proved in Section 4, that locally, in some open neighborhood $U\left(M_{h}^{r}\right) \subset M^{2 n}$ of the integral submanifold $M_{h}^{r} \subset M^{2 n}$ one can find by algebraic-analytical means just $n-r \in \mathbb{Z}_{+}$independent vector fields $\tilde{F}_{j} \in K(\mathcal{G}) / K\left(\mathcal{G}_{h}\right) \cap \Gamma\left(U\left(M_{h}^{r}\right)\right), j=\overline{1, n-r}$, satisfying condition (4.3). Since each vector field $\tilde{F}_{j} \in K(\mathcal{G}) / K\left(\mathcal{G}_{h}\right), j=\overline{1, n-r}$, is generated by an invariant $\tilde{H}_{j} \in \mathcal{D}\left(U\left(M_{h}^{r}\right)\right), j=\overline{1, n-r}$, it follows readily from (4.3) that

$$
\left\{\tilde{H}_{i}, \tilde{H}_{j}\right\}=0
$$

for all $i, j=\overline{1, n-r}$. Thus, in an open neighborhood $U\left(M_{h}^{r}\right)$ there exist just $n-r$ invariants in addition to $\tilde{H}_{j} \in \mathcal{D}\left(U\left(M_{h}^{r}\right)\right), j=\overline{1, n-r}$, all of which are in involution. Denote as before this new set of invariants as $\mathcal{G}_{\tau}$, keeping in mind that $\operatorname{dim} \mathcal{G}_{\tau}=r+(n-r)=n \in \mathbb{Z}_{+}$. Whence, in an open neighborhood $U\left(M_{h}^{r}\right) \subset M^{2 n}$ we have constructed the set $\mathcal{G}_{\tau}$ of just $n=1 / 2 \operatorname{dim} M^{2 n}$ invariants commuting with each other, thereby guaranteeing via the abelian Liouville-Arnold theorem its local complete integrability by quadratures. Consequently, there exists locally a mapping $\pi_{\tau}: M_{h, \tau}^{k} \rightarrow M^{2 n}$, where $M_{h, \tau}^{k}:=U\left(M_{h}^{r}\right) \cap M_{\tau}^{k}$ is the integral submanifold of the differential system $K(\mathcal{G})$, and one can therefore describe the behavior of integrable vector fields on the reduced manifold $\bar{M}_{h, \tau}^{2(n-r)}:=M_{h, \tau}^{k-r} / G_{h}$. For global integrability properties of a given set $\mathcal{G}$ of invariants on $\left(M^{2 n}, \omega^{(2)}\right)$, satisfying the Mishchenko-Fomenko condition (4.1), it is necessary to have the additional set of invariants $\tilde{H}_{j} \in \mathcal{D}\left(U\left(M_{h}^{r}\right)\right), j=\overline{1, n-r}$, extended from $U\left(M_{h}^{r}\right)$ to the entire phase space $M^{2 n}$. This problem evidently depends on the existence of extensions of vector fields $\tilde{F}_{j} \in \Gamma\left(U\left(M_{h}^{r}\right)\right), j=\overline{1, n-r}$, from the neighborhood $U\left(M_{h}^{r}\right) \subset M^{2 n}$ to the whole phase space $M^{2 n}$. On the other hand, as stated before, the existence of such a continuation depends intimately on the properties of the complexified differential system $K^{\mathbb{C}}(\mathcal{G}) / K^{\mathbb{C}}\left(\mathcal{G}_{h}\right)$, which has a nondegenerate complex metric $\omega\left(\tilde{K}^{\mathbb{C}}\right): T\left(\bar{M}_{h, \tau}^{2(n-r)}\right)^{\mathbb{C}} \times T\left(\bar{M}_{h, \tau}^{2(n-r)}\right)^{\mathbb{C}} \rightarrow \mathbb{C}$, induced by the symplectic structure $\omega^{(2)} \in \Lambda^{2}\left(M^{2 n}\right)$. This point can be clarified further by using the notion [24-27] of a Kähler manifold and some of the associated constructions presented above. Namely, 
consider the local isomorphism $T\left(\bar{M}_{h, \tau}^{2(n-r)}\right)^{\mathbb{C}} \simeq T\left({ }^{\mathbb{C}} \bar{M}_{h, \tau}^{n-r}\right)$, where ${ }^{\mathbb{C}} \bar{M}_{h, \tau}^{n-r}$ is the complex $(n-r)$-dimensional local integral submanifold of the complexified differential system $K^{\mathbb{C}}(\mathcal{G}) / K^{\mathbb{C}}\left(\mathcal{G}_{h}\right)$. This means that the space $T\left(\bar{M}_{h, \tau}^{2(n-r)}\right)$ is endowed with the standard almost complex structure

$$
J: T\left(\bar{M}_{h, \tau}^{2(n-r)}\right) \rightarrow T\left(\bar{M}_{h, \tau}^{2(n-r)}\right), \quad J^{2}=-1,
$$

such that the 2-form $\omega(\tilde{K}):=\operatorname{Im} \omega\left(\tilde{K}^{\mathbb{C}}\right) \in \Lambda^{2}\left(\bar{M}_{h, \tau}^{2(n-r)}\right)$ induced from the above metric on $T\left({ }^{\mathbb{C}} \bar{M}_{h, \tau}^{n-r}\right)$ is closed, that is $d \omega(\tilde{K})=0$. If this is the case, the almost complex structure on the manifold $T\left(\bar{M}_{h, \tau}^{2(n-r)}\right)$ is said to be integrable. Define the proper complex manifold ${ }^{\mathbb{C}} \bar{M}_{h, \tau}^{n-r}$, on which one can then define global vector fields $\tilde{F}_{j} \in K(\mathcal{G}) / K\left(\mathcal{G}_{h}\right), j=\overline{1, n-r}$, which are being sought for the involutive algebra $\mathcal{G}_{\tau}$ of invariants on $M^{2 n}$ to be integrable by quadratures via the abelian LiouvilleArnold theorem. Thus the following theorem can be obtained.

Theorem 16. A nonabelian set $\mathcal{G}$ of invariants on the symplectic space $M^{2 n} \simeq$ $T^{*}\left(\mathbb{R}^{n}\right)$, satisfying the Mishchenko-Fomenko condition (2.9), admits algebraic-analytical integration by quadratures for the integral submanifold imbedding $\pi_{h}: M_{h}^{r} \rightarrow$ $M^{2 n}$ if the corresponding complexified reduced manifold ${ }^{\mathbb{C}} \bar{M}_{h, \tau}^{n-r} \simeq \bar{M}_{h, \tau}^{2(n-r)}=M_{h, \tau}^{k-r} / G_{h}$ of the differential system $K^{\mathbb{C}}(\mathcal{G}) / K^{\mathbb{C}}\left(\mathcal{G}_{h}\right)$ is Kählerian with respect to the standard almost complex structure (5.1) and the nondegenerate complex metric $\omega\left(\tilde{K}^{\mathbb{C}}\right)$ : $T\left(\bar{M}_{h, \tau}^{2(n-r)}\right)^{\mathbb{C}} \times T\left(\bar{M}_{h, \tau}^{2(n-r)}\right)^{\mathbb{C}} \rightarrow \mathbb{C}$ induced by the symplectic structure $\omega^{(2)} \in \Lambda^{2}\left(M^{2 n}\right)$ is integrable, that is $d \operatorname{Im} \omega\left(\tilde{K}^{\mathbb{C}}\right)=0$.

Theorem 16 shows, in particular, that nonabelian Liouville-Arnold integrability by quadratures does not in general imply integrability via the abelian Liouville-Arnold theorem; it actually depends on certain topological obstructions associated with the Lie algebra structure of invariants $\mathcal{G}$ on the phase space $M^{2 n}$. We hope to explore this intriguing problem in another place.

\section{SupPlement}

In this section we consider some examples of investigation of integral submanifold imbedding mappings for abelian Liouville-Arnold integrable Hamiltonian systems on $T^{*}\left(\mathbb{R}^{2}\right)$.

7.1. The Henon-Heiles system. This flow is governed by the Hamiltonian

$$
H_{1}=\frac{1}{2} p_{1}^{2}+\frac{1}{2} p_{2}^{2}+q_{1} q_{2}^{2}+\frac{1}{3} q_{1}^{3}
$$


on the canonically symplectic phase space $M^{4}=T^{*}\left(\mathbb{R}^{2}\right)$ with the symplectic structure

$$
\omega^{(2)}=\sum_{j=1}^{2} d p_{j} \wedge d q_{j}
$$

As is well known, there exists the following additional invariant that commutes with (7.1):

$$
H_{2}=p_{1} p_{2}+1 / 3 q_{2}^{3}+q_{1}^{2} q_{2}
$$

that is $\left\{H_{1}, H_{2}\right\}=0$ on the entire space $M^{4}$.

Take a regular element $h \in \mathcal{G}:=\left\{H_{j}: M^{4} \rightarrow \mathbb{R}, j=\overline{1,2}\right\}$, with fixed values $h\left(H_{j}\right)=h_{j} \in \mathbb{R}, j=\overline{1,2}$. Then the integral submanifold

$$
M_{h}^{2}:=\left\{(q, p) \in M^{4}: h\left(H_{j}\right)=h_{j} \in \mathbb{R}, j=\overline{1,2}\right\},
$$

if compact and connected, is diffeomorphic to the standard torus $\mathbb{T}^{2} \simeq \mathbb{S}^{1} \times \mathbb{S}^{1}$ owing to the Liouville-Arnold theorem, and one can find cyclic (separable) coordinates $\mu_{j} \in \mathbb{S}^{1}, j=\overline{1,2}$, on the torus such that the symplectic structure (7.2) will take the form:

$$
\omega^{(2)}=\sum_{j=1}^{2} d w_{j} \wedge d \mu_{j},
$$

where the conjugate variables $w_{j} \in T^{*}\left(\mathbb{S}^{1}\right), j=\overline{1,2}$, on $M_{h}^{2}$ depend only on the corresponding variables $\mu_{j} \in \mathbb{S}_{j}^{1}, j=\overline{1,2}$. In this case it is evident that the evolution along $M_{h}^{2}$ will be separable and representable by means of quasi-periodic functions of the evolution parameters.

To show this, recall that the fundamental determining equations (3.34) based on the 1 -forms $\bar{h}_{j}^{(1)} \in \Lambda\left(M_{h}^{2}\right), j=\overline{1,2}$, satisfy the identity

$$
\sum_{j=1}^{2} d H_{j} \wedge_{j} \bar{h}_{j}^{(1)}=\sum_{j=1}^{2} d p_{j} \wedge d q_{j}
$$

Here

$$
\bar{h}_{j}^{(1)}=\sum_{k=1}^{2} \bar{h}_{j k}(q, p) d q_{k}
$$

where $j=\overline{1,2}$. Substituting (7.7) into (7.6), one obtains

$$
\bar{h}_{1}^{(1)}=\frac{p_{1} d q_{1}}{p_{1}^{2}-p_{2}^{2}}+\frac{p_{2} d q_{2}}{p_{1}^{2}-p_{2}^{2}}, \quad \bar{h}_{2}^{(1)}=\frac{p_{2} d q_{1}}{p_{21}^{2}-p_{1}^{2}}+\frac{p_{1} d q_{2}}{p_{1}^{2}-p_{2}^{2}} .
$$

On the other hand, the following implication holds on $M_{h}^{2} \subset M^{4}$ :

$$
\alpha_{h}^{(1)}=\sum_{j=1}^{2} w_{j}\left(\mu_{j} ; h\right) d \mu_{j} \quad \Longrightarrow \quad \sum_{j=1}^{2} p_{j} d q_{j}:=\alpha^{(1)}
$$


where we have assumed that the integral submanifold $M_{h}^{2}$ admits the local coordinates in the base manifold $\mathbb{R}^{2}$ endowed with the canonical 1-form $\alpha_{h}^{(1)} \in \Lambda\left(M_{h}^{2}\right)$ as given in (7.9). Thus, making use of the imbedding $\pi_{h}: M_{h}^{2} \rightarrow T^{*}\left(\mathbb{R}^{2}\right)$ in the form

$$
q_{j}=q_{j}(\mu ; h), \quad p_{j}=p_{j}(\mu ; h),
$$

$j=\overline{1,2}$, one readily finds that the equalities

$$
p_{j}=\sum_{k=1}^{2} w_{k}\left(\mu_{k} ; h\right) \partial \mu_{k} / \partial q_{j}
$$

hold for $j=\overline{1,2}$ on the entire integral submanifold $M_{h}^{2}$.

Substituting (7.11) into (7.8) and using the characteristic relationships (3.34), one obtains after simple but cumbersome calculations the following differential-algebraic expressions:

$$
\partial q_{1} / \partial \mu_{1}-\partial q_{2} / \partial \mu_{1}=0, \quad \partial q_{1} / \partial \mu_{2}+\partial q_{2} / \partial \mu_{2}=0,
$$

whose simplest solutions are

$$
q_{1}=\left(\mu_{1}+\mu_{2}\right) / 2, \quad q_{2}=\left(\mu_{1}-\mu_{2}\right) / 2 .
$$

Using expressions (7.11) one finds that

$$
p_{1}=w_{1}+w_{2}, \quad p_{2}=w_{1}-w_{2},
$$

where

$$
w_{1}=\sqrt{h_{1}+h_{2}-4 / 3 \mu_{1}^{3}}, \quad w_{2}=\sqrt{h_{1}-h_{2}-4 / 3 \mu_{2}^{3}} .
$$

Consequently, one obtains the separable [15] Hamiltonian functions (7.1) and (7.3) in the vicinity of the cotangent space $T^{*}\left(M_{h}^{2}\right)$ :

$$
h_{1}=\frac{1}{2} w_{1}^{2}+\frac{1}{2} w_{2}^{2}+\frac{2}{3}\left(\mu_{1}^{3}+\mu_{2}^{3}\right), \quad h_{2}=\frac{1}{2} w_{1}^{2}-\frac{1}{2} w_{2}^{2}+\frac{2}{3}\left(\mu_{1}^{3}-\mu_{2}^{3}\right),
$$

which generate the following separable motions on $M_{h}^{2} \subset T^{*}\left(\mathbb{R}^{2}\right)$ :

$$
\begin{aligned}
& d \mu_{1} / d t:=\partial h_{1} / \partial w_{1}=\sqrt{h_{1}+h_{2}-4 / 3 \mu_{1}^{3}}, \\
& d \mu_{2} / d t:=\partial h_{1} / \partial w_{2}=\sqrt{h_{1}-h_{2}-4 / 3 \mu_{2}^{3}}
\end{aligned}
$$

for the Hamiltonian (7.1), and

$$
\begin{aligned}
& d \mu_{1} / d x:=\partial h_{2} / \partial w_{1}=\sqrt{h_{1}+h_{2}-4 / 3 \mu_{1}^{3}}, \\
& d \mu_{2} / d t:=\partial h_{1} / \partial w_{2}=-\sqrt{h_{1}-h_{2}-4 / 3 \mu_{2}^{3}}
\end{aligned}
$$

for the Hamiltonian (7.3), where $x, t \in \mathbb{R}$ are the corresponding evolution parameters. 
Analogously, one can show that there exists [28,29] a similar to (7.13) and (7.14) integral submanifold imbedding for the following integrable modified Henon-Heiles involutive system:

$$
\begin{gathered}
H_{1}=\frac{1}{2} p_{1}^{2}+\frac{1}{2} p_{2}^{2}+q_{1} q_{2}^{2}+\frac{16}{3} q_{1}^{3}, \\
H_{2}=9 p_{2}^{4}+36 q_{1} p_{2}^{2} q_{2}^{2}-12 p_{1} p_{2} q_{2}^{3}-2 q_{2}^{4}\left(q_{2}^{2}+6 q_{1}^{2}\right),
\end{gathered}
$$

where $\left\{H_{1}, H_{1}\right\}=0$ on the entire phase space $M^{4}=T^{*}\left(\mathbb{R}^{2}\right)$.

Based on considerations similar to the above, one can deduce the following [29] expressions:

$$
\begin{aligned}
& q_{1}=-\frac{1}{4}\left(\mu_{1}+\mu_{2}\right)-\frac{3}{8}\left(\frac{w_{1}+w_{2}}{\mu_{1}-\mu_{2}}\right)^{2}, \\
& q_{2}^{2}=-2 \sqrt{h_{2}} /\left(\mu_{1}-\mu_{2}\right), \quad w_{1}=\sqrt{2 / 3 \mu_{1}^{3}-4 / 3 \sqrt{h_{2}}-8 h_{1}}, \\
& p_{1}=\frac{1}{2 \sqrt{-6\left(\mu_{1}+\mu_{2}+4 q\right)}}\left[\frac{-2 \sqrt{h_{2}}}{\mu_{1}-\mu_{2}}-\mu_{1} \mu_{2}+4\left(\mu_{1}+\mu_{2}\right) q_{1}+32 q_{1}^{2}\right], \\
& p_{2}=\sqrt{h_{2}}\left(\mu_{1}+\mu_{2}+4 q_{1}\right) /\left(3\left(\mu_{1-} \mu_{2}\right)\right), \quad w_{2}=\sqrt{2 / 3 \mu_{2}^{3}+4 / 3 \sqrt{h_{2}}-8 h_{1}},
\end{aligned}
$$

thereby solving explicitly the problem of finding the corresponding integral submanifold imbedding $\pi_{h}: M_{h}^{2} \rightarrow T^{*}\left(\mathbb{R}^{2}\right)$ that generates separable flows in the variables $(\mu, w) \in T^{*}\left(M_{h}^{2}\right)$.

7.2. A truncated 4-dimensional Focker-Plank Hamiltonian system on $T^{*}\left(\mathbb{R}^{2}\right)$ and its integrability by quadratures. Consider the following dynamical system on the canonically symplectic phase space $T^{*}\left(\mathbb{R}^{2}\right)$ :

$$
\left.\begin{array}{l}
d q_{1} / d t=p_{1}+\alpha\left(q_{1}+p_{2}\right)\left(q_{2}+p_{1}\right), \quad d q_{2} / d t=p_{2}, \\
d p_{1} / d t=-\left(q_{1}+p_{2}\right)-\alpha\left[q_{2} p_{1}+1 / 2\left(p_{1}^{2}+p_{2}^{2}+q_{2}^{2}\right)\right], \\
d p_{2} / d t=-\left(q_{2}+p_{1}\right),
\end{array}\right\}=K_{1}(q, p),
$$

where $K_{1}: T^{*}\left(\mathbb{R}^{2}\right) \rightarrow T\left(T^{*}\left(\mathbb{R}^{2}\right)\right)$ is the corresponding vector field on $T^{*}\left(\mathbb{R}^{2}\right) \ni$ $(q, p), t \in \mathbb{R}$ is an evolution parameter, called a truncated four-dimensional FockerPlank flow. It is easy to verify that functions $H_{j}: T^{*}\left(\mathbb{R}^{2}\right) \rightarrow \mathbb{R}, j=\overline{1,2}$, where

$$
H_{1}=1 / 2\left(p_{1}^{2}+p_{2}^{2}+q_{1}^{2}\right)+q_{1} p_{2}+\alpha\left(q_{1}+p_{2}\right)\left[q_{2} p_{1}+1 / 2\left(p_{1}^{2}+p_{2}^{2}+q_{2}^{2}\right)\right]
$$

and

$$
H_{2}=1 / 2\left(p_{1}^{2}+p_{2}^{2}+q_{2}^{2}\right)+q_{2} p_{1}
$$

are functionally independent invariants with respect to the flow (7.21). Moreover, the invariant (7.22) is the Hamiltonian function for (7.21), that is the relationship

$$
i_{K_{1}} \omega^{(2)}=-d H_{1}
$$


holds on $T^{*}\left(\mathbb{R}^{2}\right)$, where the symplectic structure $\omega^{(2)} \in \Lambda^{2}\left(T^{*}\left(\mathbb{R}^{2}\right)\right)$ is given as follows:

$$
\omega^{(2)}:=d\left(\operatorname{pr}^{*} \alpha^{(1)}\right)=\sum_{j=1}^{2} d p_{j} \wedge d q_{j},
$$

with $\alpha^{(1)} \in \Lambda^{1}\left(\mathbb{R}^{2}\right)$ to be the canonical Liouville form on $\mathbb{R}^{2}$ :

$$
\alpha^{(1)}(q ; p)=\sum_{j=1}^{2} p_{j} d q_{j}
$$

for any $(q, p) \in T^{*}\left(\mathbb{R}^{2}\right) \simeq \Lambda^{1}\left(\mathbb{R}^{2}\right)$.

The invariants (7.22) and (7.23) commute evidently with each other subject to the associated Poisson bracket on $T^{*}\left(\mathbb{R}^{2}\right)$, i. e., $\left\{H_{1}, H_{2}\right\}=0$. Thereby, owing to the abelian Liouville-Arnold theorem [1,2], the dynamical system (7.21) is completely integrable by quadratures on $T^{*}\left(\mathbb{R}^{2}\right)$, and we can apply the scheme devised in Section 2 to the commuting invariants (7.22) and (7.23) subject to the symplectic structure (7.25). One easily calculates that

$$
\omega^{(2)}=\sum_{i=1}^{2} d H_{i} \wedge h_{i}^{(1)},
$$

where the corresponding 1 -forms $\pi_{h}^{*} h_{i}^{(1)}:=\bar{h}_{i}^{(1)} \in \Lambda^{1}\left(M_{h}^{2}\right), i=\overline{1,2}$, are given as

$$
\begin{aligned}
\bar{h}_{1}^{(1)} & =\frac{p_{2} d q_{1}-\left(p_{1}+q_{2}\right) d q_{2}}{p_{1} p_{2}-\left(p_{1}+q_{2}\right)\left(q_{1}+p_{2}\right)-\alpha h_{2}\left(p_{1}+q_{2}\right)}, \\
\bar{h}_{2}^{(1)} & =\frac{-\left[\left(q_{1}+p_{2}\right)\left(1+\alpha p_{2}\right)+\alpha h_{2}\right] d q_{1}+\left(p_{1}+\alpha\left[h_{2}+\left(q_{2}+p_{1}\right)\left(q_{1}+p_{2}\right)\right]\right) d q_{2}}{p_{1} p_{2}-\left(q_{2}+p_{1}\right)\left(\alpha h_{2}+q_{1}+p_{2}\right)},
\end{aligned}
$$

and an invariant submanifold $M_{h}^{2} \subset T^{*}\left(\mathbb{R}^{2}\right)$ is defined as

$$
M_{h}^{2}:=\left\{(q, p) \in T^{*}\left(\mathbb{R}^{2}\right): H_{i}(q, p)=h_{i} \in \mathbb{R}, i=\overline{1,2}\right\}
$$

for some parameters $h \in \mathbb{R}^{2}$ and based now on expressions (7.28), and (3.38) one can easily construct functions $\bar{P}_{i j}(w ; h), i, j=\overline{1,2}$, in (3.53), defined on $T^{*}\left(M_{h}^{2}\right) \simeq$ $T^{*}\left(\otimes_{j=1}^{2} \mathrm{~S}_{j}^{1}\right)$ subject to the integral submanifold imbedding mapping $\pi_{h}: M_{h}^{2} \rightarrow$ $T^{*}\left(\mathbb{R}^{2}\right)$ in coordinates $\mu \in \otimes_{j=1}^{2} \mathbb{S}_{j}^{1} \subset \otimes_{j=1}^{2} \Gamma_{h}^{(j)}$, which we do not write in detail due to their long and cumbersome form. Having applied then the criterion (3.55), we arrive at the following compatibility relations subject to the mappings $q:\left(\otimes_{j=1}^{2} \mathbb{S}_{j}^{1}\right) \times \mathbb{R}^{2} \rightarrow$ 


$$
\begin{gathered}
\mathbb{R}^{2} \text { and } p:\left(\otimes_{j=1}^{2} \mathbb{S}_{j}^{1}\right) \times \mathbb{R}^{2} \rightarrow T_{q}^{*}\left(\mathbb{R}^{2}\right): \\
\partial q_{1} / \partial \mu_{1}-\partial q_{2} / \partial \mu_{2}=0, \quad w_{1} \partial \mathcal{L}_{\mu} / \partial w_{1}-w_{2} \partial \mathcal{L}_{\mu} / \partial w_{2}=0, \\
\partial^{2} q_{1} / \partial \mu_{2} \partial h_{2}+\partial^{2} w_{2} / \partial \mu_{2} \partial h_{2}=0, \\
\partial w_{1} / \partial h_{1}\left(\partial q_{1} / \partial h_{1}\right)=\partial w_{2} / \partial h_{1}\left(\partial q_{2} / \partial h_{1}\right), \\
w_{1} \partial w_{1} / \partial h_{1}-w_{2} \partial w_{2} / \partial h_{2}=0, \\
\partial\left(w_{1} \partial w_{1} / \partial h_{2}\right) / \partial h_{2}-\alpha^{2} \partial q_{1} / \partial \mu_{1}=0, \ldots
\end{gathered}
$$

and so on, subject to variables $\mu \in \otimes_{j=1}^{2} \mathbb{S}_{j}^{1}$ and $h \in \mathbb{R}^{2}$. Solving equations like (7.29), one can find right away that the expressions

$$
\begin{gathered}
p_{1}=w_{1}, \quad p_{2}=w_{2}, \\
q_{1}=c_{1}+\mu_{1}-w_{2}\left(\mu_{2} ; h\right), \quad q_{2}=c_{2}+\mu_{2}-w_{1}\left(\mu_{1} ; h\right), \\
\mathcal{L}_{\mu}(h)=-w_{1} w_{2},
\end{gathered}
$$

where $c_{j}\left(h_{1}, h_{2}\right) \in \mathbb{R}^{1}, j=\overline{1,2}$, are constant, hold on $T^{*}\left(M_{h}^{2}\right)$, giving rise to the following Picard- Fuchs type equations in the form (3.56):

$$
\begin{aligned}
\partial w_{1}\left(\mu_{1} ; h\right) / \partial h_{1}=1 / w_{1}, & & \partial w_{1}\left(\mu_{1} ; h\right) / \partial h_{2} & =\alpha^{2} h_{2} / w_{1}, \\
\partial w_{2}\left(\mu_{2} ; h\right) / \partial h_{1}=0, & & \partial w_{2}\left(\mu_{2} ; h\right) / \partial h_{2} & =1 / w_{2} .
\end{aligned}
$$

The Picard-Fuchs equations (7.31) can be easily integrated by quadratures as follows:

$$
w_{1}^{2}+k_{1}\left(\mu_{1}\right)-\alpha^{2} h_{2}-2 h_{1}=0, \quad w_{2}^{2}+k_{2}\left(\mu_{2}\right)-2 h_{2}=0,
$$

where $k_{j}: \mathbb{S}_{j}^{1} \rightarrow \mathbb{C}, j=\overline{1,2}$, are still unknown functions. For them to be determined explicitly, it is necessarily to substitute (7.30) into expressions (7.22) and (7.23), making use of (7.32), which amounts to the following results:

$$
k_{1}=\mu_{1}^{2}, \quad k_{2}=\mu_{2}^{2}
$$

under the condition that $c_{1}=-\alpha h_{2}, c_{2}=0$. Thereby, we have constructed, owing to (7.32), the corresponding algebraic curves $\Gamma_{h}^{(j)}, j=\overline{1.2},(3.41)$ in the explicit form:

$$
\begin{gathered}
\Gamma_{h}^{(1)}:=\left\{\left(\lambda, w_{1}\right): w_{1}^{2}+\lambda^{2}-\alpha^{2} h_{2}^{2}-2 h_{1}=0\right\}, \\
\Gamma_{h}^{(2)}:=\left\{\left(\lambda, w_{2}\right): w_{2}^{2}+\lambda^{2}-2 h_{2}=0\right\},
\end{gathered}
$$

where $\left(\lambda, w_{j}\right) \in \mathbb{C}, j=\overline{1,2}$, and $h \in \mathbb{R}^{2}$ are arbitrary parameters. Making use now of expressions (7.35) and (7.30), one can construct in explicit form the integral submanifold imbedding mapping $\pi_{h}: M_{h}^{2} \rightarrow T^{*}\left(\mathbb{R}^{2}\right)$ for the flow (7.21):

$$
\begin{aligned}
& q_{1}=\mu_{1}-\sqrt{2 h_{2}-\mu_{2}^{2}}-\alpha h_{2}^{2}, \quad p_{1}=w_{1}\left(\mu_{1} ; h\right), \\
& q_{2}=\mu_{2}-\sqrt{2 h_{1}-\alpha^{2} h_{2}^{2}-\mu_{1}^{2}}, \quad p_{2}=w_{2}\left(\mu_{2} ; h\right),
\end{aligned}
$$


where $(\mu, w) \in \otimes_{j=1}^{2} \Gamma_{h}^{(j)}$. As mentioned before in Section 2, formulas (7.35) together with explicit expressions (3.40) make it possible right away to find solutions to the truncated Focker-Plank flow (7.21) by quadratures, thereby completing its integrability.

\section{ACKNOWLEDGEMENTS}

The authors are cordially thankful to Prof. Boris A. Kupershmidt (Space Institute, University of Tennessee, Tullahoma, USA) for many important suggestions and critical comments concerning the exposition of some statements in the article. One of the authors (A. P.) is also deeply thankful to Prof. Péter Nagy (Institute of Mathematics and Informatics, Debrecen University, Hungary) and Prof. Miklós Rontó (Institute of Mathematics, University of Miskolc, Hungary) for the kind invitation to visit them during the 2002 winter semester and deliver talks on differential-geometric aspects of integrable dynamical systems and for the many stimulating discussions of the problems treated in this article.

\section{REFERENCES}

[1] Abraham, R. and Marsden, J.: Foundations of Mechanics, Cummings Co., USA, 1978.

[2] Mishchenko, A. S. and Fomenko, A. T.: Generalized Liouville method of integrating Hamiltonian systems, Funkcional. Anal. i Prilozhen., 12 (1978), No. 2, 46-56.

[3] Cartan, É.: Leçons sur invariants integraux, Hermann, Paris, 1971.

[4] Arnold, V. I.: Mathematical Methods of Classical Mechanics, Springer, NY, 1978.

[5] Fomenko, A. T.: Differential Geometry and Topology. Supplementary Chapters, Moscow University Press, 1983.

[6] Marsden, J. and Weinstein A.: Reduction of symplectic manifolds with symmetry, Reports on Math. Phys., 5 (1974), No. 1, 121-130.

[7] Zakrzewski, S.: Induced representations and induced Hamiltonian actions, Journ. of Geometry and Physics, 3 (1986), No. 2, 11-219.

[8] Kummer, M.: On the construction of the reduced phase space of a Hamilton system with symmetry, Indiana Univ. Math. Journal, 30 (1981), No. 2, 281-291.

[9] SATzer, W. J., JR.: Canonical reduction of mechanical systems invariant under abelian group actions with an application to celestial mechanics, Indiana Univ. Math. Journal, 26 (1977), No. 5, 951-976.

[10] Souriau, J. M.: Structures des systèmes dynamiques, Dunod, Paris, 1970.

[11] Prykarpatsky, Ya. A., Samoilenko, A. M., and Blackmore, D. L.: Imbedding of integral submanifolds and associated adiabatic invariants of slowly perturbed integrable Hamiltonian systems, Reports on Mathem. Physics, 44 (1999), Nos. 1\&2, 171-182

[12] Basarab-Horwath, P.: Integrability by quadratures for systems of involutive vector fields, Ukrain. Math. J., 43(1992), No. 10, 1236-1242.

[13] Kopych, M., Prykarpatsky, Ya., AND Samulyak, R.: Adiabatic invariants of a generalized HenonHeiles Hamiltonian system and structure of the chaotic motion, Dop. Nats. Akad. Ukr. (1997), No. 2, 32-36.

[14] Ankiewicz, A. And Pask, C.: The complete Whittaker theorem for two-dimensional integrable systems and its application, Journal of Physics, A, 16 (1983), No. 18, 4203-4208. 
[15] Rauch-Wojciechowski, S. and Tsiganov, A. V.: Quiasi point separation of variables for the Henon-Heiles systems and a system with quadratic potential, Journal of Physics, A, 29 (1996), No. 23, 7769-7778.

[16] Prytula, M., Prykarpatsky, A., and Myкytiuk, I.: Fundamentals of the Theory of DifferentialGeometric Structures and Dynamical Systems Kiev, Ministry of Education Publ., 1988 (in Ukrainian).

[17] Prykarpatsky, A. and Myкytiuk, I.: Algebraic Integrability of Nonlinear Dynamical Systems on Manifolds: Classical and Quantum Aspects, Kluwer, Dordrecht, 1998.

[18] Françoise, J. P.: Monodromy and the Kowalewskaya top, Asterisque, 150/151 (1987), 87-108; Arnold's formula for algebraically completely integrable systems, Bull. Amer. Math. Soc., 17 (1987), 301-303.

[19] Deligne, P.: Equations différentielles a points singulairs, Lecture Notes in Math., Springer, 1970.

[20] Zverovich, E.: Boundary problems of the theory of analytical functions in Hölder classes on Riemannian surfaces, Russian Math. Surveys, 26 (1971), No. 1, 113-176 (in Russian).

[21] Blaszak, M.: Multi-Hamiltonian Dynamical Systems, Springer, 1998.

[22] Prykarpatsky, A. and Myкytiuk, I.: Algebraic Aspects of Integrable Dynamical Systems on Manifolds, Kiev, Naukova Dumka Publ., 1987 (in Russian).

[23] Blackmore, D., Prykarpatsky, Ya., and Samulyak, R.: On the Lie invariant geometric objects, generated by integrable ideals in Grassmann algebra, J. Nonl. Math. Physics, 5 (1998), No. 1, 54-67.

[24] Wells, R. O.: Differential Analysis on Complex Manifolds, Prentice-Hall, Englewood Cliff., NJ, 1973.

[25] Newlander, A. ANd Nirenberg, L.: Complex analytical coordinates in almost-complex manifolds, Ann. of Mathematics, 65 (1957), 391-404.

[26] Hörmander, L.: An Introduction to Complex Analysis in Several Variables, Van Nostrand Reinhold Publ. Co., 1986.

[27] Kobayashi, S. and Nomizu, K.: Foundations of Differential Geometry, John Wiley and Sons, NY, Vol. 1, 1963; Vol. 2, 1969.

[28] Salerno, M., Enolski, V. Z., and Leykin, D. V.: Canonical transformation between integrable Henon-Heiles systems, Phys. Rev. E, 49 (1994), No. 6, 5897-58899.

[29] Ravoson, V., Gavrilov, L., and Caloz, R.: Separability and Lax pairs for Henon-Heiles system, J. Mathem. Physics, 34 (1993), No. 6, 2385-2393.

[30] Roekaerts, D. and Scwarz, F.: Painlevé analysis, Yoshida's theorems and direct methods in the search for integrable Hamiltonians, J. Phys. A: Mat. Gen., 20 (1987), L127-L133.

[31] HöRMANDER, L.: Course at Lund University, 1974.

[32] DuistermaAt, J. J.: On global action-angle coordinates, Comm. Pure and Appl. Math., 33 (1980), 687-706.

[33] Kozlov, V. V. and Kolesnikov, N. N.: On dynamics theorems, Prykl. Mathem. and Mechanics, 42 (1978), No. 1, 28-33.

[34] Nieknoroshev, N. N.: Action-angle variables and their generalization, Trudy Mosk. Matem. Obshchestva, 26 (1972), 181-198.

\section{Authors' Addresses}

\section{Y. A. Prykarpatsky:}

Institute of Mathematics, NAS of Ukraine, 01601 Kyiv, Ukraine

Current address: Department of Applied Mathematics, AGH University of Science and Technology, 30-059 Kraków, Poland

E-mail address: yarchyk@imath.kiev.ua 


\section{A. M. Samoilenko:}

Institute of Mathematics, NAS of Ukraine, 01601 Kyiv, UKraine

E-mail address: sam@imath.kiev.ua

\section{L. Blackmore:}

Department of Mathematical Sciences, NJIT, Newark, NJ 07102, USA

E-mail address: deblac@m.njit.edu, deblac@chaos.njit.edu

\section{A. K. Prykarpatsky:}

Institute for Applied Problems of Mechanics and Mathematics, NAS of Ukraine, 79601 Lviv, UKRAINE

Current address: Department of Applied Mathematics, AGH University of Science and Technology, 30059 Kraków, Poland

E-mail address: pryk.anat@ua.fm, prykanat@cybergal.com 